\title{
The eclectic flavor symmetry of the $\mathbb{Z}_{2}$ orbifold
}

\author{
Alexander Baur, ${ }^{a, c}$ Moritz Kade, ${ }^{a}$ Hans Peter Nilles, ${ }^{b}$ Saúl Ramos-Sánchez ${ }^{a, c}$ and \\ Patrick K.S. Vaudrevange ${ }^{a}$ \\ ${ }^{a}$ Physik Department Tr5, Technische Universität München, \\ James-Franck-Straße 1, Garching 85748, Germany \\ ${ }^{b}$ Bethe Center for Theoretical Physics and Physikalisches Institut der Universität Bonn, \\ Nussallee 12, Bonn 53115, Germany \\ ${ }^{c}$ Instituto de Física, Universidad Nacional Autónoma de México, \\ POB 20-364, Ciudad de México 01000, México \\ E-mail: alexander.baur@tum.de, moritz.kade@tum.de, \\ nilles@th.physik.uni-bonn.de, ramos@fisica.unam.mx, \\ patrick. vaudrevange@tum.de
}

ABSTRACT: Modular symmetries naturally combine with traditional flavor symmetries and $\mathcal{C P}$, giving rise to the so-called eclectic flavor symmetry. We apply this scheme to the twodimensional $\mathbb{Z}_{2}$ orbifold, which is equipped with two modular symmetries $\operatorname{SL}(2, \mathbb{Z})_{T}$ and $\mathrm{SL}(2, \mathbb{Z})_{U}$ associated with two moduli: the Kähler modulus $T$ and the complex structure modulus $U$. The resulting finite modular group is $\left(\left(S_{3} \times S_{3}\right) \rtimes \mathbb{Z}_{4}\right) \times \mathbb{Z}_{2}$ including mirror symmetry (that exchanges $T$ and $U$ ) and a generalized $\mathcal{C P}$-transformation. Together with the traditional flavor symmetry $\left(D_{8} \times D_{8}\right) / \mathbb{Z}_{2}$, this leads to a huge eclectic flavor group with 4608 elements. At specific regions in moduli space we observe enhanced unified flavor symmetries with as many as 1152 elements for the tetrahedral shaped orbifold and $\langle T\rangle=\langle U\rangle=\exp \left(\frac{\pi \mathrm{i}}{3}\right)$. This rich eclectic structure implies interesting (modular) flavor groups for particle physics models derived form string theory.

Keywords: Compactification and String Models, Discrete Symmetries, Field Theories in Higher Dimensions, Superstrings and Heterotic Strings

ARXIV EPRINT: 2008.07534 


\section{Contents}

1 Introduction 1

$2 \quad$ Flavor from outer automorphisms of the $\mathbb{Z}_{2}$ space group 2

3 Flavor from modular symmetries $\quad 6$

4 Local flavor unification $\quad 10$

4.1 The generic $\mathbb{T}^{2} / \mathbb{Z}_{2}$ orbifold 13

$\begin{array}{ll}4.2 \text { The tetrahedron with }\langle U\rangle=e^{\frac{\pi \mathrm{i}}{3}} & 14\end{array}$

$\begin{array}{ll}4.3 \text { The raviolo with }\langle U\rangle=\mathrm{i} & 16\end{array}$

$\begin{array}{lll}4.4 & \mathbb{T}^{2} / \mathbb{Z}_{2} \text { orbifolds with } \mathcal{C P} \text {-enhancement } & 17\end{array}$

5 Conclusions and outlook $\quad 17$

$\begin{array}{ll}\text { A Narain lattice } & 19\end{array}$

$\begin{array}{ll}\text { A.1 Orbifolds defined by the Narain space group } & 19\end{array}$

A.2 Outer automorphisms of the $\mathbb{Z}_{2}$ Narain space group 20

A.3 Transformation of $\mathbb{Z}_{2}$ twisted strings $\quad 21$

$\begin{array}{lll}\text { A.4 Details on mirror symmetry } & 22\end{array}$

B How to classify the outer automorphisms of a space group $\quad 23$

C Irreducible representations of twisted matter fields $\quad 24$

\section{Introduction}

In the present paper we further explore the eclectic flavor picture in the framework of orbifold compactifications of string theory. The eclectic flavor symmetry is the maximal discrete symmetry that can arise from the nontrivial combination of traditional flavor symmetry and modular flavor symmetry [1]. In this approach, the benefits of finite modular symmetries, uncovered by ref. [2] and further developed for example in refs. [3-6], are merged with the appeal of traditional flavor symmetries (see e.g. [7]), possibly improving [8] their predictability [9]. Up to now the detailed analysis has concentrated on two-dimensional orbifolded tori where the complex structure modulus $U$ is fixed geometrically to allow for the specific orbifold twist of the torus [10,11]. Aspects of the embedding of the two-tori into six-dimensional compactified space have been discussed in ref. [12] and have shown to be especially relevant for the discussion of $R$-symmetries. To capture the full eclectic picture one has to consider also those orbifolded tori, where the complex structure modulus is not fixed. This will certainly lead to a richer eclectic structure, as modular 
transformations now act nontrivially on both, the Kähler modulus $T$ and the complex structure modulus $U$. As a first step in this analysis, we consider the $\mathbb{T}^{2} / \mathbb{Z}_{2}$ orbifold that allows the full action of $\mathrm{SL}(2, \mathbb{Z})_{T}$ and $\mathrm{SL}(2, \mathbb{Z})_{U}$. This captures all the qualitative aspects of the eclectic flavor picture and can be used as building block for the general discussion in the six-dimensional case.

Flavor symmetries of the $\mathbb{T}^{2} / \mathbb{Z}_{2}$ orbifold strongly depend on the values of the moduli. First, we discuss in section 2 the case for generic values of both $T$ and $U$ that gives rise to the traditional flavor symmetry (which leaves the moduli invariant). This leads to the traditional flavor group $\left(D_{8} \times D_{8}\right) / \mathbb{Z}_{2} \cong[32,49]$, see ref. [13]. The numbers [32,49] correspond to a unique identifier, assigned by the computer program GAP [14], where the first number (32) gives the order of the group. The finite modular group of the $\mathbb{T}^{2} / \mathbb{Z}_{2}$ orbifold is derived in section 3 and turns out to be $[144,115]$, the multiplicative closure of mirror symmetry and the $S_{3} \times S_{3}$ finite modular groups arising from $\operatorname{SL}(2, \mathbb{Z})_{T}$ and $\operatorname{SL}(2, \mathbb{Z})_{U}$. If we further include a $\mathcal{C P}$-like modular transformation $[3,10,11,15]$, this group is enhanced to $[288,880]$. Thus, by combining the traditional flavor group and the finite modular group we are led to an eclectic flavor group with maximally 2304 elements (without $\mathcal{C P}$ ) and 4608 elements (including $\mathcal{C P}$ ).

Typically, only a subgroup of the eclectic flavor group is linearly realized and its size depends on the values of the moduli. This leads to an enhancement of the traditional flavor group $\left(D_{8} \times D_{8}\right) / \mathbb{Z}_{2}$ at specific points and hypersurfaces in moduli space: the so-called mechanism of local flavor unification, which is discussed in section 4 . There are two specific configurations of the $\mathbb{T}^{2} / \mathbb{Z}_{2}$ orbifold that deserve special attention: the raviolo (at $\langle U\rangle=\mathrm{i}$, see figure 1) and the tetrahedron (at $\langle U\rangle=\exp \left(\frac{\pi \mathrm{i}}{3}\right)$, see figure 5$)$. There, we observe a further enhancement of the unified flavor symmetry. The largest linearly realized group is found at $\langle T\rangle=\langle U\rangle=\exp \left(\frac{\pi \mathrm{i}}{3}\right)$ and turns out to be [1152,157463], which includes mirror symmetry and $\mathcal{C P}$. The landscape of unified flavor symmetries (with $\mathcal{C P}$ ) is illustrated in figure 7. Even for an orbifold as simple as $\mathbb{T}^{2} / \mathbb{Z}_{2}$ we find amazingly large flavor groups. In section 5 we shall summarize our results and give an outlook on future research tasks. These should include a full implementation of the automorphy factors (in the spirit of refs. $[12,16])$ and a road-map towards an embedding in the six-dimensional case. Some technical results are relegated to three appendices.

\section{Flavor from outer automorphisms of the $\mathbb{Z}_{2}$ space group}

In order to specify the two-dimensional $\mathbb{T}^{2} / \mathbb{Z}_{2}$ orbifold, we first define the geometrical space group $S$. The space group consists of elements $g=\left(\theta^{k}, e n\right) \in S$, with $k \in\{0,1\}$, that act on the coordinates $y \in \mathbb{R}^{2}$ of the extra dimensions as

$$
y \stackrel{g}{\mapsto} g y:=\theta^{k} y+e n,
$$

where the $\mathbb{Z}_{2}$ twist $\theta$ is given by $\theta=-\mathbb{1}_{2}$ and $n=\left(n_{1}, n_{2}\right)^{\mathrm{T}} \in \mathbb{Z}^{2}$ are called winding numbers. One can easily see from eq. (2.1) that two space group elements multiply as

$$
\left(\theta^{k}, e n\right)\left(\theta^{k^{\prime}}, e n^{\prime}\right)=\left(\theta^{k+k^{\prime}}, e n+\theta^{k} e n^{\prime}\right) .
$$



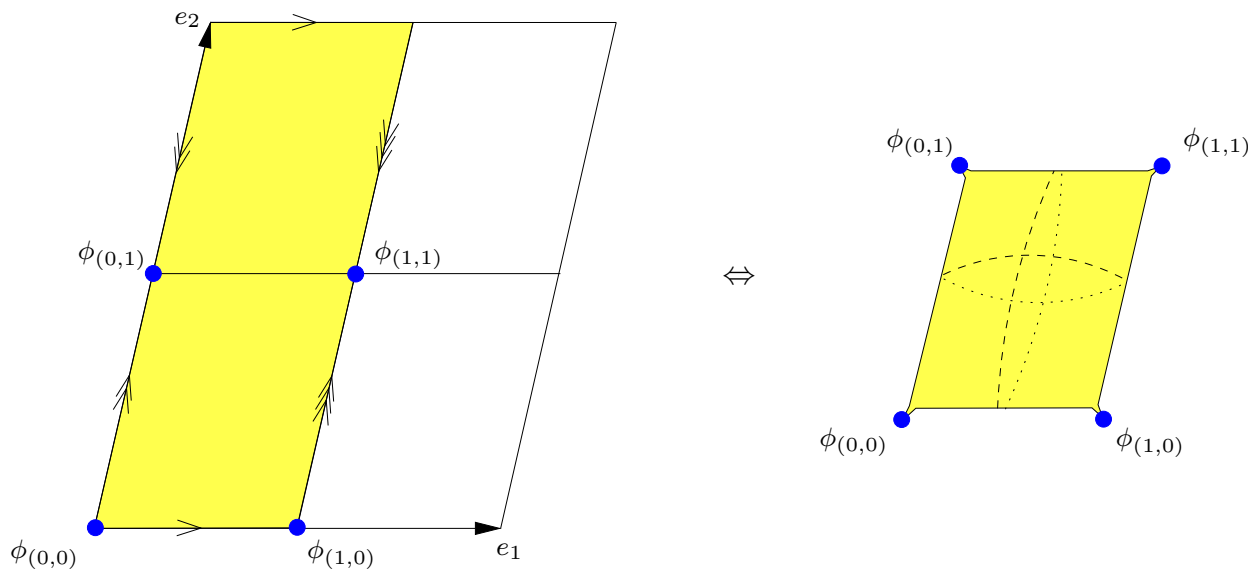

Figure 1. The $\mathbb{T}^{2} / \mathbb{Z}_{2}$ orbifold. Left: the two-torus $\mathbb{T}^{2}$ is defined by a two-dimensional lattice, spanned by the vectors $e_{1}$ and $e_{2}$. The $\mathbb{Z}_{2}$ orbifold twist $\theta=-\mathbb{1}_{2}$ maps the fundamental domain of $\mathbb{T}^{2}$ to the fundamental domain of the orbifold, depicted in yellow. The orbifold action $y \mapsto$ $\theta^{k} y+e n$ has four inequivalent fixed points, indicated by blue bullets. Localized at these fixed points, there are four (left-chiral) twisted strings $\left(\phi_{(0,0)}, \phi_{(1,0)}, \phi_{(0,1)}, \phi_{(1,1)}\right)$. The boundaries of the yellow fundamental domain are identified according to the arrows. Right: after identifying the boundaries of the fundamental domain, the $\mathbb{T}^{2} / \mathbb{Z}_{2}$ orbifold has a pillow-like shape with the four fixed points at the corners of the pillow (or raviolo).

The $2 \times 2$ matrix $e$ (called the geometrical vielbein) consists of two columns $e_{1}$ and $e_{2}$. These vectors have to be linearly independent, so that they span a two-dimensional lattice that defines a two-torus $\mathbb{T}^{2}$. Then, the $\mathbb{T}^{2} / \mathbb{Z}_{2}$ orbifold $\mathbb{O}$ is defined as a quotient space

$$
\mathbb{O}:=\frac{\mathbb{R}^{2}}{S}, \quad \text { where } y \sim y^{\prime} \quad \text { if there exists } \quad g \in S \text { such that } y^{\prime}=g y,
$$

i.e. points $y, y^{\prime} \in \mathbb{R}^{2}$ in extra dimensions are identified if they are related by the orbifold action with some space group element $g \in S$. This yields a reduced fundamental domain of the orbifold, see figure 1.

Closed strings on $\mathbb{O}$ are defined by boundary conditions for the string world-sheet degrees of freedom [17-19], with world-sheet coordinates $\tau$ and $\sigma$. Concentrating on the world-sheet bosons $y(\tau, \sigma)$ that describe two extra spatial dimensions $y$, a boundary condition for a closed string is given by

$$
y(\tau, \sigma+1)=g y(\tau, \sigma)=\theta^{k} y(\tau, \sigma)+e n,
$$

where $g=\left(\theta^{k}, e n\right) \in S$ with $k \in\{0,1\}$ is the so-called constructing element of the string. In fact, inequivalent strings correspond not only to constructing elements $g \in S$ but to their conjugacy classes $[g]:=\left\{f^{-1} g f \mid f \in S\right\}$, since $y(\tau, \sigma)$ and $f y(\tau, \sigma)$ are identified on the orbifold for all $f \in S$. If $k=0$ in eq. (2.4), the string is called untwisted and lives in the bulk of the orbifold. In this case, it can still wind around the two-torus depending on its winding numbers $n \in \mathbb{Z}^{2}$. If $k=1$, the string is called a twisted string. Then, its center of mass is given by the fixed point $y_{g}$ of $g \in S$. In more detail, $y_{g}$ denotes the solution of the fixed point equation $g y_{g}=\theta y_{g}+e n=y_{g}$. For $g=(\theta, e n) \in S$, it reads 
$y_{g}=\frac{1}{2}$ en. Furthermore, the internal momentum of a twisted string vanishes such that a twisted string stays localized at its fixed point $y_{g}$.

For the $\mathbb{T}^{2} / \mathbb{Z}_{2}$ orbifold, there are four conjugacy classes of twisted strings with constructing elements $g=(\theta, e n)=\left(\theta, n_{1} e_{1}+n_{2} e_{2}\right) \in S$. They are given by

$$
\left[\left(\theta, n_{1} e_{1}+n_{2} e_{2}\right)\right]=\left\{\left(\theta,\left(n_{1}+2 \tilde{n}_{1}\right) e_{1}+\left(n_{2}+2 \tilde{n}_{2}\right) e_{2}\right) \in S \mid \tilde{n}_{1}, \tilde{n}_{2} \in \mathbb{Z}\right\} .
$$

Hence, winding numbers $n_{1}$ and $n_{2}$ of twisted strings are only defined modulo two and we can choose $n_{1}, n_{2} \in\{0,1\}$. We denote the four twisted matter fields associated with the four conjugacy classes of twisted strings by $\phi_{\left(n_{1}, n_{2}\right)}$, i.e.

$$
\phi_{\left(n_{1}, n_{2}\right)} \Leftrightarrow \quad \Leftrightarrow \quad\left[\left(\theta, n_{1} e_{1}+n_{2} e_{2}\right)\right] \quad \text { for } \quad n_{1}, n_{2} \in\{0,1\} .
$$

Moreover, the matter field $\phi_{\left(n_{1}, n_{2}\right)}$ is localized at the fixed point $y_{g}=\frac{1}{2}\left(n_{1} e_{1}+n_{2} e_{2}\right)$ in $\mathbb{O}$, as illustrated in figure 1.

Discrete flavor symmetries of the effective four-dimensional field theory from strings on orbifolds find their origin in the outer automorphisms of the so-called Narain space group $[10,11]$. Since the Narain construction of strings on orbifolds is rather technical, we refer here only to a short discussion in appendix A and to the literature [20-22]. Still, one can gain some insights by considering the outer automorphisms of the geometrical space group $S$ (instead of the Narain space group). An outer automorphism of $S$ is given by a transformation $h=(\sigma, e t) \notin S$, such that

$$
g \stackrel{h}{\longmapsto} h^{-1} g h \stackrel{!}{\in} S \quad \text { for all } g \in S,
$$

see e.g. ref. [11] for a similar discussion in the case of a $\mathbb{T}^{2} / \mathbb{Z}_{3}$ orbifold.

For the $\mathbb{T}^{2} / \mathbb{Z}_{2}$ orbifold, the outer automorphisms of the geometrical $\mathbb{Z}_{2}$ space group that leave the moduli unaltered are generated by two translations,

$$
h_{1}:=\left(\mathbb{1}_{2}, \frac{1}{2} e_{1}\right) \quad \text { and } \quad h_{2}:=\left(\mathbb{1}_{2}, \frac{1}{2} e_{2}\right) .
$$

In the absence of nontrivial discrete Wilson lines [19], they give rise to symmetries of the full string construction, see appendix A for the corresponding Narain construction. Then, one is interested in the action $\rho_{\mathbf{4}}(h)$ of a (geometrical) transformation $h \notin S$ on the four twisted matter fields

$$
\left(\begin{array}{l}
\phi_{(0,0)} \\
\phi_{(1,0)} \\
\phi_{(0,1)} \\
\phi_{(1,1)}
\end{array}\right) \stackrel{h}{\longmapsto} \rho_{\mathbf{4}}(h)\left(\begin{array}{c}
\phi_{(0,0)} \\
\phi_{(1,0)} \\
\phi_{(0,1)} \\
\phi_{(1,1)}
\end{array}\right) .
$$

On the level of constructing elements, one already realizes that, for example, the translation $h_{1}$ acts as

$$
\begin{aligned}
\left(\theta, n_{1} e_{1}+n_{2} e_{2}\right) \stackrel{h_{1}}{\longmapsto}\left(\mathbb{1}_{2}, \frac{-1}{2} e_{1}\right)\left(\theta, n_{1} e_{1}+n_{2} e_{2}\right)\left(\mathbb{1}_{2}, \frac{1}{2} e_{1}\right) \\
=\left(\theta,\left(n_{1}-1\right) e_{1}+n_{2} e_{2}\right) .
\end{aligned}
$$


Hence, the transformation $h_{1}$ interchanges $\phi_{\left(0, n_{2}\right)}$ and $\phi_{\left(1, n_{2}\right)}$ for $n_{2} \in\{0,1\}$, see figure 2 . Similarly, one can show that $h_{2}$ exchanges the twisted matter fields $\phi_{\left(n_{1}, 0\right)}$ and $\phi_{\left(n_{1}, 1\right)}$ for $n_{1} \in\{0,1\}$. This geometrical intuition can be confirmed by a direct computation on twisted string states (see appendix A). We thus find

$$
\rho_{4}\left(h_{1}\right)=\left(\begin{array}{llll}
0 & 1 & 0 & 0 \\
1 & 0 & 0 & 0 \\
0 & 0 & 0 & 1 \\
0 & 0 & 1 & 0
\end{array}\right) \quad \text { and } \quad \rho_{4}\left(h_{2}\right)=\left(\begin{array}{llll}
0 & 0 & 1 & 0 \\
0 & 0 & 0 & 1 \\
1 & 0 & 0 & 0 \\
0 & 1 & 0 & 0
\end{array}\right) .
$$

These transformations generate a $\mathbb{Z}_{2} \times \mathbb{Z}_{2}$ Abelian flavor symmetry.

In addition, there are string selection rules restricting the ability of strings on orbifolds to join and split [23]. The associated symmetry can be determined in two ways: i) as the Abelianization of the space group $S$ [24], or ii) as additional outer automorphisms of the Narain space group. Combined with the geometrical transformations $h_{1}$ and $h_{2}$, which exchange orbifold fixed points pairwise, these string selection rules yield a non-Abelian flavor symmetry as follows: in the $\mathbb{T}^{2} / \mathbb{Z}_{2}$ orbifold, the string selection rules give rise to a $\mathbb{Z}_{2} \times \mathbb{Z}_{2} \times \mathbb{Z}_{2}$ symmetry, under which twisted matter fields transform as

$$
\begin{aligned}
& \phi_{\left(n_{1}, n_{2}\right)} \stackrel{h_{3}}{\longmapsto}(-1)^{n_{1}} \phi_{\left(n_{1}, n_{2}\right)} \quad \Rightarrow \quad \rho_{4}\left(h_{3}\right)=\left(\begin{array}{cccc}
1 & 0 & 0 & 0 \\
0 & -1 & 0 & 0 \\
0 & 0 & 1 & 0 \\
0 & 0 & 0 & -1
\end{array}\right), \\
& \phi_{\left(n_{1}, n_{2}\right)} \stackrel{h_{4}}{\longmapsto}(-1)^{n_{2}} \phi_{\left(n_{1}, n_{2}\right)} \quad \Rightarrow \quad \rho_{4}\left(h_{4}\right)=\left(\begin{array}{cccc}
1 & 0 & 0 & 0 \\
0 & 1 & 0 & 0 \\
0 & 0 & -1 & 0 \\
0 & 0 & 0 & -1
\end{array}\right) \text {, } \\
& \phi_{\left(n_{1}, n_{2}\right)} \stackrel{h_{5}}{\longmapsto}-\phi_{\left(n_{1}, n_{2}\right)} \quad \Rightarrow \quad \rho_{4}\left(h_{5}\right)=\left(\begin{array}{cccc}
-1 & 0 & 0 & 0 \\
0 & -1 & 0 & 0 \\
0 & 0 & -1 & 0 \\
0 & 0 & 0 & -1
\end{array}\right) .
\end{aligned}
$$

Here, $h_{3}$ and $h_{4}$ are connected to the $\mathbb{Z}_{2} \times \mathbb{Z}_{2}$ space group selection rule for twisted strings, while $h_{5}$ is associated with the $\mathbb{Z}_{2}$ point group selection rule. Then, as first shown in ref. [13], the full traditional flavor group (without $R$-symmetry) of the $\mathbb{T}^{2} / \mathbb{Z}_{2}$ orbifold, at a generic point in moduli space, is generated by the transformations (2.11) and (2.12), resulting in

$$
\frac{\left(D_{8} \times D_{8}\right)}{\mathbb{Z}_{2}} \cong[32,49]
$$

Here, $D_{8} \cong[8,3]$ denotes the dihedral group of order 8 (sometimes also called $D_{4}$ using a different naming convention). Furthermore, the first $D_{8}$ factor in eq. (2.13) is generated by $h_{1}$ and $h_{3}$. This $D_{8}$ is associated with the $e_{1}$ direction of the orbifold, cf. ref. [25]. The second $D_{8}$ in eq. (2.13) is generated by $h_{2}$ and $h_{4}$ and is associated with $e_{2}$. Note that 


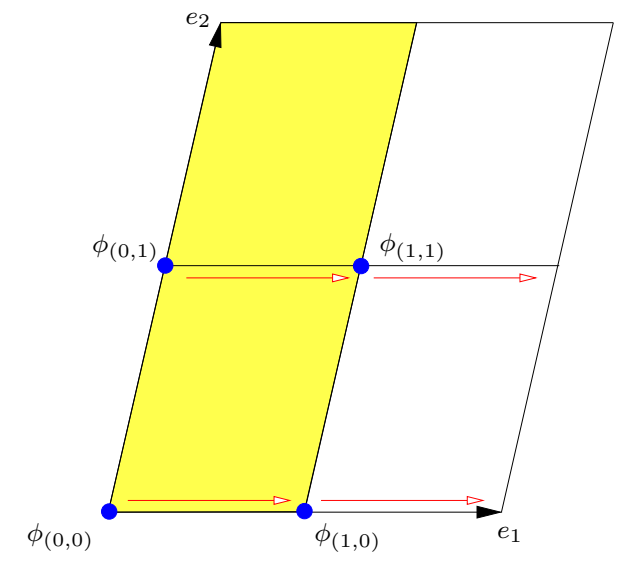

(a) Transformation $h_{1}=\left(\mathbb{1}_{2}, \frac{1}{2} e_{1}\right)$.

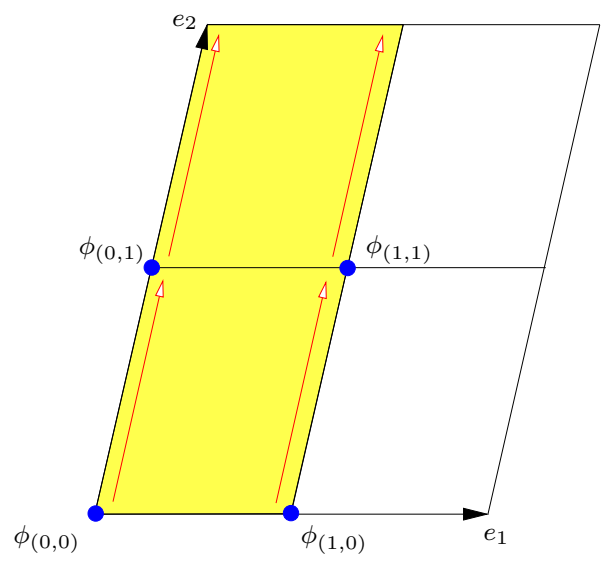

(b) Transformation $h_{2}=\left(\mathbb{1}_{2}, \frac{1}{2} e_{2}\right)$.

Figure 2. Actions of the outer automorphisms $h_{1}$ and $h_{2}$ of the $\mathbb{T}^{2} / \mathbb{Z}_{2}$ orbifold space group on the four twisted matter fields $\left(\phi_{(0,0)}, \phi_{(1,0)}, \phi_{(0,1)}, \phi_{(1,1)}\right)$. (a) Under the geometrical translation $h_{1}:=$ $\left(\mathbb{1}_{2}, \frac{1}{2} e_{1}\right)$, matter fields get interchanged as $\phi_{\left(0, n_{2}\right)} \leftrightarrow \phi_{\left(1, n_{2}\right)}$ for $n_{2} \in\{0,1\}$. (b) The geometrical translation $h_{2}:=\left(\mathbb{1}_{2}, \frac{1}{2} e_{2}\right)$ interchanges matter fields as $\phi_{\left(n_{1}, 0\right)} \leftrightarrow \phi_{\left(n_{1}, 1\right)}$ for $n_{1} \in\{0,1\}$.

the transformation $h_{5}$ in eq. (2.12c), linked to the point group selection rule, is not an independent generator of the traditional flavor group eq. (2.13) as it can be written as

$$
\rho_{\mathbf{4}}\left(h_{5}\right)=\left(\rho_{\mathbf{4}}\left(h_{1}\right) \rho_{\mathbf{4}}\left(h_{3}\right)\right)^{2}=\left(\rho_{\mathbf{4}}\left(h_{2}\right) \rho_{\mathbf{4}}\left(h_{4}\right)\right)^{2} .
$$

This identity gives rise to the $\mathbb{Z}_{2}$ quotient in eq. (2.13). Moreover, the four twisted matter fields $\left(\phi_{(0,0)}, \phi_{(1,0)}, \phi_{(0,1)}, \phi_{(1,1)}\right)^{\mathrm{T}}$ build a four-dimensional, irreducible, and faithful representation of the traditional flavor group eq. (2.13).

Even though we have discussed the origin of the traditional flavor symmetry $\left(D_{8} \times\right.$ $\left.D_{8}\right) / \mathbb{Z}_{2}$ based on the outer automorphisms of the geometrical $\mathbb{Z}_{2}$ space group, the results can be confirmed using the full Narain approach, see appendices A and B. Even more, the full Narain space group and its outer automorphisms reveal a common origin of all discrete symmetries for strings on orbifolds, giving rise to the eclectic flavor symmetry that consists of traditional flavor, modular, $\mathcal{C P}$ and $R$-symmetries. In the next section, we will analyze in detail the modular symmetries and their finite modular groups that arise in the $\mathbb{T}^{2} / \mathbb{Z}_{2}$ orbifold.

\section{Flavor from modular symmetries}

In general, the deformations of a two-dimensional torus used to compactify a string theory can be parameterized by a complex structure modulus $U$ and a Kähler modulus $T$. The complex structure modulus $U$ can be interpreted geometrically as the shape of the torus, while the Kähler modulus $T$ gives the overall size of the torus and the value of the anti-symmetric $B$-field background. Moreover, a toroidal compactification exhibits several 
symmetries that act nontrivially on these moduli (see e.g. ref. [11]):

$$
\begin{array}{lll}
U \stackrel{\hat{C}_{\mathrm{S}}}{\longmapsto}-\frac{1}{U} & \text { and } & T \stackrel{\hat{C}_{\mathrm{S}}}{\longmapsto} T, \\
U \stackrel{\hat{C}_{\mathrm{T}}}{\longmapsto} U+1 & \text { and } & T \stackrel{\hat{C}_{\mathrm{T}}}{\longmapsto} T, \\
U \stackrel{\hat{K}_{\mathrm{S}}}{\longmapsto} U & \text { and } & T \stackrel{\hat{K}_{\mathrm{S}}}{\longmapsto}-\frac{1}{T}, \\
U \stackrel{\hat{K}_{\mathrm{T}}}{\longmapsto} U & \text { and } & T \stackrel{\hat{K}_{\mathrm{T}}}{\longmapsto} T+1 .
\end{array}
$$

These transformations are actually not defined on the level of the moduli but on the level of outer automorphisms of the Narain space group, see appendix A.2. Then, $\hat{C}_{\mathrm{S}}$ and $\hat{C}_{\mathrm{T}}$ generate a modular group $\mathrm{SL}(2, \mathbb{Z})_{U}$ associated with the complex structure modulus $U$, while $\hat{K}_{\mathrm{S}}$ and $\hat{K}_{\mathrm{T}}$ are the generators of another factor $\mathrm{SL}(2, \mathbb{Z})_{T}$ associated with the Kähler modulus $T$. Note that the two factors of $\operatorname{SL}(2, \mathbb{Z})$ share a common element: $\mathrm{C}:=$ $\left(\hat{C}_{\mathrm{S}}\right)^{2}=\left(\hat{K}_{\mathrm{S}}\right)^{2}$. Even though $\mathrm{C}$ acts trivially on both moduli, it can in principle still act nontrivially on matter fields, see refs. $[8,11]$ (and also ref. [26]). In addition, there are two special transformations

$$
\begin{array}{lll}
U \stackrel{\hat{M}}{\longmapsto} T & \text { and } & T \stackrel{\hat{M}}{\longmapsto} U, \\
U \stackrel{\hat{\Sigma}_{*}}{\longmapsto}-\bar{U} & \text { and } & T \stackrel{\hat{\Sigma}_{*}}{\longmapsto}-\bar{T} .
\end{array}
$$

The former is the origin of the so-called mirror symmetry that interchanges $\operatorname{SL}(2, \mathbb{Z})_{U}$ and $\operatorname{SL}(2, \mathbb{Z})_{T}$, while the latter induces a $\mathcal{C} \mathcal{P}$-like transformation, see refs. $[10,15]$ and [3].

In principle, performing an orbifold of a torus can stabilize some moduli geometrically. Consequently, some of the symmetry transformations generated by eqs. (3.1) and (3.2) can be broken by the orbifolding. For example, in the case of a $\mathbb{T}^{2} / \mathbb{Z}_{3}$ orbifold sector the $U$ modulus needs to be stabilized, e.g. at $\langle U\rangle=\exp \left(\frac{2 \pi \mathrm{i}}{3}\right)$, and the unbroken modular symmetry after $\mathbb{Z}_{3}$ orbifolding is generated by $\hat{K}_{\mathrm{S}}, \hat{K}_{\mathrm{T}}$, the $R$-symmetry $\hat{C}_{\mathrm{S}} \hat{C}_{\mathrm{T}}$ and the $\mathcal{C P}$-like transformation $\hat{K}_{*}:=\hat{C}_{\mathrm{S}} \hat{C}_{\mathrm{T}} \hat{C}_{\mathrm{S}} \hat{\Sigma}_{*}$ (i.e. by those modular transformations that leave $\langle U\rangle=\exp \left(\frac{2 \pi \mathrm{i}}{3}\right)$ invariant). In contrast, the $\mathbb{T}^{2} / \mathbb{Z}_{2}$ orbifold with $\theta=-\mathbb{1}_{2}$ is equipped with both moduli: the complex structure modulus $U$ and the Kähler modulus $T$. Each of them remains associated with its own unbroken modular group, $\operatorname{SL}(2, \mathbb{Z})_{U}$ and $\operatorname{SL}(2, \mathbb{Z})_{T}$ for $U$ and $T$, respectively. Moreover, the transformations (3.2) remain symmetries after the torus has been modded out by the $\mathbb{Z}_{2}$ orbifold action $\theta=-\mathbb{1}_{2}$. Hence, the $\mathbb{T}^{2} / \mathbb{Z}_{2}$ orbifold gives a simple example of a string setup with multiple modular symmetries (see e.g. ref. [27]), with the extension by mirror symmetry eq. (3.2a) that interchanges both moduli.

As shown in refs. $[12,16]$, the modular group $\operatorname{SL}(2, \mathbb{Z})_{U}$ of the complex structure modulus acts geometrically on the compact dimensions. In particular, the $\operatorname{SL}(2, \mathbb{Z})_{U}$ generators $\hat{C}_{\mathrm{S}}$ and $\hat{C}_{\mathrm{T}}$ act on the $\mathbb{T}^{2}$ basis vectors $e_{i}$ according to

$$
e_{1} \stackrel{\hat{C}_{\mathrm{S}}}{\longrightarrow} e_{1}^{\prime}=-e_{2}, \quad e_{2} \stackrel{\hat{C}_{\mathrm{S}}}{\longrightarrow} e_{2}^{\prime}=e_{1}, \quad \text { and } \quad e_{1} \stackrel{\hat{C}_{\mathrm{T}}}{\longrightarrow} e_{1}^{\prime}=e_{1}, \quad e_{2} \stackrel{\hat{C}_{\mathrm{T}}}{\longrightarrow} e_{2}^{\prime}=e_{1}+e_{2} .
$$


This can be confirmed easily by e.g. considering each two-dimensional vector $e_{i}$ as a complex number, i.e. $e_{1}, e_{2} \in \mathbb{C}$, and setting $U=\frac{e_{2}}{e_{1}}$, see ref. [28]. Then, eq. (3.3) reproduces eqs. (3.1a) and (3.1b).

Next, we are interested in the action of $\operatorname{SL}(2, \mathbb{Z})_{U}$ on the four twisted matter fields $\phi_{\left(n_{1}, n_{2}\right)}$, i.e. for $\gamma_{U} \in \mathrm{SL}(2, \mathbb{Z})_{U}$ we want to identify $\rho_{\mathbf{4}}\left(\gamma_{U}\right)$ defined as

$$
\left(\begin{array}{l}
\phi_{(0,0)} \\
\phi_{(1,0)} \\
\phi_{(0,1)} \\
\phi_{(1,1)}
\end{array}\right) \stackrel{\gamma_{U}}{\longmapsto}\left(\begin{array}{c}
\phi_{(0,0)}^{\prime} \\
\phi_{(1,0)}^{\prime} \\
\phi_{(0,1)}^{\prime} \\
\phi_{(1,1)}^{\prime}
\end{array}\right)=\rho_{\mathbf{4}}\left(\gamma_{U}\right)\left(\begin{array}{l}
\phi_{(0,0)} \\
\phi_{(1,0)} \\
\phi_{(0,1)} \\
\phi_{(1,1)}
\end{array}\right)
$$

without taking the automorphy factors $\left(c_{U} U+d_{U}\right)^{n_{U}}$ of $\gamma_{U}$ with modular weight $n_{U}$ into account. Then, one can use the geometrical $\mathrm{SL}(2, \mathbb{Z})_{U}$ transformations (3.3) as illustrated in figure 3 in order to obtain the matrix representations of the modular $\mathrm{S}$ and $\mathrm{T}$ transformations, given by $\hat{C}_{\mathrm{S}}$ and $\hat{C}_{\mathrm{T}}$, for the four twisted matter fields $\phi_{\left(n_{1}, n_{2}\right)}$. The results read

$$
\rho_{\mathbf{4}}\left(\hat{C}_{\mathrm{S}}\right)=\left(\begin{array}{llll}
1 & 0 & 0 & 0 \\
0 & 0 & 1 & 0 \\
0 & 1 & 0 & 0 \\
0 & 0 & 0 & 1
\end{array}\right) \quad \text { and } \quad \rho_{\mathbf{4}}\left(\hat{C}_{\mathrm{T}}\right)=\left(\begin{array}{llll}
1 & 0 & 0 & 0 \\
0 & 1 & 0 & 0 \\
0 & 0 & 0 & 1 \\
0 & 0 & 1 & 0
\end{array}\right) .
$$

One can check that these representation matrices $\rho_{\mathbf{4}}\left(\hat{C}_{\mathrm{S}}\right)$ and $\rho_{\mathbf{4}}\left(\hat{C}_{\mathrm{T}}\right)$ generate a so-called finite modular group $S_{3} \cong[6,1]$ : even though the symmetry is $\operatorname{SL}(2, \mathbb{Z})_{U}$, twisted matter fields transform under $\mathrm{SL}(2, \mathbb{Z})_{U}$ in unitary representations of $S_{3}$. We denote this factor by $S_{3}^{U}$ as it is associated with the complex structure modulus $U$.

Also the mirror symmetry $\hat{M}$ has a non-trivial action on twisted matter fields. It turns out that it can be represented by the matrix

$$
\rho_{4}(\hat{M})=\frac{1}{\sqrt{2}}\left(\begin{array}{cccc}
0 & 0 & -1 & 1 \\
0 & 0 & 1 & 1 \\
1 & -1 & 0 & 0 \\
-1 & -1 & 0 & 0
\end{array}\right)
$$

see appendix A.4. Interestingly, one can verify easily that

$$
\left(\rho_{4}(\hat{M})\right)^{2}=-\mathbb{1}_{4}
$$

Hence, $\rho_{\mathbf{4}}(\hat{M})$ is of order 4. Moreover, eq. (3.7) shows that, although $\hat{M}^{2}$ acts trivially on the moduli, it acts nontrivially on twisted matter fields: in fact, $\left(\rho_{\mathbf{4}}(\hat{M})\right)^{2}$ acts like the traditional flavor transformation $\rho_{4}\left(h_{5}\right)=-\mathbb{1}_{4}$ associated with the point group selection rule, see eq. (2.12c). Consequently, the finite modular group can not be disentangled from the traditional flavor group completely: the element $\hat{M}^{2}$ belongs to both groups. The situation is similar to the $\mathbb{T}^{2} / \mathbb{Z}_{3}$ orbifold, where the modular $\mathrm{S}$ transformation squared equals a traditional flavor transformation: $\left(\hat{C}_{\mathrm{S}}\right)^{2}=\left(\hat{K}_{\mathrm{S}}\right)^{2}=\mathrm{C}$ from $\Delta(54)$, see ref. [11]. 
Then, we can translate the finite modular group $S_{3}^{U}$ of the complex structure modulus $U$ to the one of the Kähler modulus $T$. Using eq. (A.13) from appendix A.2, we obtain

$$
\begin{aligned}
& \rho_{\mathbf{4}}\left(\hat{K}_{\mathrm{S}}\right):=\rho_{\mathbf{4}}(\hat{M}) \rho_{\mathbf{4}}\left(\hat{C}_{\mathrm{S}}\right) \rho_{\mathbf{4}}(\hat{M})^{-1}=\frac{1}{2}\left(\begin{array}{cccc}
1 & 1 & 1 & 1 \\
1 & 1 & -1 & -1 \\
1 & -1 & 1 & -1 \\
1 & -1 & -1 & 1
\end{array}\right), \\
& \rho_{\mathbf{4}}\left(\hat{K}_{\mathrm{T}}\right):=\rho_{\mathbf{4}}(\hat{M}) \rho_{\mathbf{4}}\left(\hat{C}_{\mathrm{T}}\right) \rho_{\mathbf{4}}(\hat{M})^{-1}=\left(\begin{array}{cccc}
-1 & 0 & 0 & 0 \\
0 & 1 & 0 & 0 \\
0 & 0 & 1 & 0 \\
0 & 0 & 0 & 1
\end{array}\right)
\end{aligned}
$$

see ref. [29]. We denote the resulting finite modular group associated with the Kähler modulus $T$ by $S_{3}^{T}$.

The final transformation from the list of generators given in eqs. (3.1) and (3.2) is the $\mathcal{C P}$-like transformation $\hat{\Sigma}_{*}$. It acts on twisted matter fields as

$$
\left(\begin{array}{l}
\phi_{(0,0)} \\
\phi_{(1,0)} \\
\phi_{(0,1)} \\
\phi_{(1,1)}
\end{array}\right) \stackrel{\Sigma_{*}}{\longmapsto}\left(\begin{array}{l}
\bar{\phi}_{(0,0)} \\
\bar{\phi}_{(1,0)} \\
\bar{\phi}_{(0,1)} \\
\bar{\phi}_{(1,1)}
\end{array}\right),
$$

where we suppress the spacetime dependencies. Hence, if one considers $\mathcal{C} \mathcal{P}$-like transformations, it is beneficial to extend the $4 \times 4$ representation matrices to $8 \times 8$ matrices acting on the eight-dimensional vector $(\Phi, \bar{\Phi})^{\mathrm{T}}$ of twisted matter fields $\Phi:=\left(\phi_{(0,0)}, \phi_{(1,0)}, \phi_{(0,1)}, \phi_{(1,1)}\right)^{\mathrm{T}}$ and their $\mathcal{C} \mathcal{P}$-partners.

In summary, the matrices $\rho_{\mathbf{4}}\left(\hat{K}_{\mathrm{S}}\right), \rho_{\mathbf{4}}\left(\hat{K}_{\mathrm{T}}\right), \rho_{\mathbf{4}}\left(\hat{C}_{\mathrm{S}}\right)$ and $\rho_{\mathbf{4}}\left(\hat{C}_{\mathrm{T}}\right)$ generate the finite modular group

$$
S_{3}^{T} \times S_{3}^{U}
$$

of order $6 \times 6=36$. Combined with the $\mathbb{Z}_{4}$ mirror element $\rho_{\mathbf{4}}(\hat{M})$, we obtain the finite modular group without $\mathcal{C P}$

$$
\left(S_{3}^{T} \times S_{3}^{U}\right) \rtimes \mathbb{Z}_{4}^{\hat{M}} \cong[144,115]
$$

which is of order $36 \times 4=144$. We observe, as a side remark, that this finite modular group is related to the group of outer automorphisms of the traditional flavor group,

$$
\operatorname{Out}\left(\left(D_{8} \times D_{8}\right) / \mathbb{Z}_{2}\right) \cong[72,40] \cong[144,115] / \mathbb{Z}_{2},
$$

where the $\mathbb{Z}_{2}$ on the right-hand side is generated by $\hat{M}^{2}$. Moreover, by including $\mathcal{C P}$ we get $[288,880] \cong[144,115] \times \mathbb{Z}_{2}$, which is the maximal finite modular group of the $\mathbb{T}^{2} / \mathbb{Z}_{2}$ orbifold.

Next, we combine the finite modular group with the traditional flavor group $\left(D_{8} \times\right.$ $\left.D_{8}\right) / \mathbb{Z}_{2}$ and construct the eclectic flavor group $G_{\text {eclectic. }}$. The traditional flavor group is a normal subgroup of $G_{\text {eclectic }}$, as expected from the general framework of eclectic flavor 


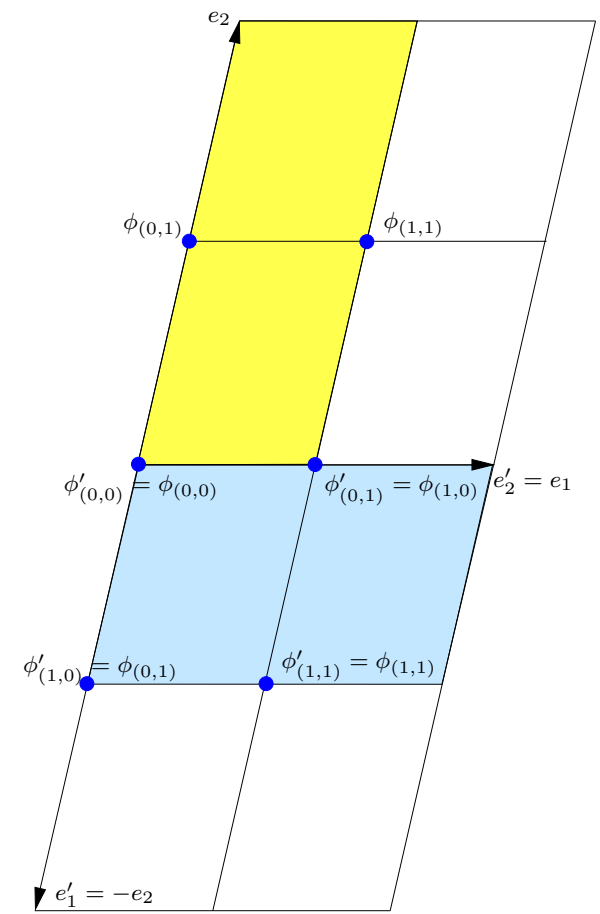

(a) Modular S transformation.

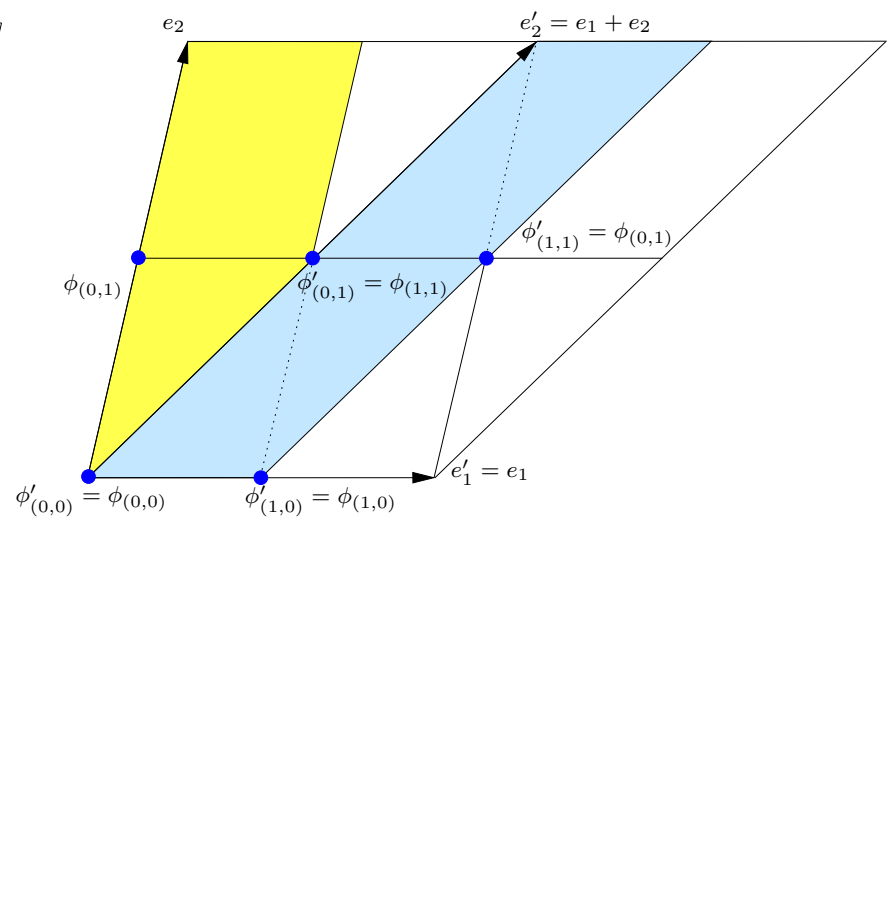

(b) Modular T transformation.

Figure 3. The action of the $\mathrm{SL}(2, \mathbb{Z})_{U}$ generators $\hat{C}_{\mathrm{S}}(\mathrm{a})$ and $\hat{C}_{\mathrm{T}}$ (b) associated with the complex structure modulus $U$ on the $\mathbb{T}^{2} / \mathbb{Z}_{2}$ orbifold. From the action of the $\operatorname{SL}(2, \mathbb{Z})_{U}$ generators on the $\mathbb{T}^{2}$ basis, eq. (3.3), the original (yellow) fundamental domain of the orbifold is mapped to an equivalent but different (blue) region. Hence, twisted matter fields $\phi_{\left(n_{1}, n_{2}\right)}$ get interchanged according to eqs. (3.4) and (3.5).

groups [1]. However, since $\left(D_{8} \times D_{8}\right) / \mathbb{Z}_{2}$ and the finite modular group share the common element $\left(\rho_{\mathbf{4}}(\hat{M})\right)^{2}=\rho_{\mathbf{4}}\left(h_{5}\right)$, the eclectic flavor group is not a semi-direct product of these two factors. In the case without $\mathcal{C P}$, the finite modular group is $\left(S_{3}^{T} \times S_{3}^{U}\right) \rtimes \mathbb{Z}_{4}^{\hat{M}}$ and $G_{\text {eclectic }}$ turns out to be of order 2304 . This order can be understood easily since $(144 \times 32) / 2=2304$ using the fact that $\left(\rho_{\mathbf{4}}(\hat{M})\right)^{2}$ belongs to both factors. As a side remark, note that all finite groups of order 2304 have been classified in ref. [30]. In appendix $\mathrm{C}$ we examine the representation $\rho_{\mathbf{4}}$ of twisted matter fields with respect to $G_{\text {eclectic }}$ and its various subgroups. In addition, if we include $\mathcal{C P}$, the eclectic flavor group gets enhanced further to a group of order 4608 .

\section{Local flavor unification}

As discussed in section 2, for generic values of the moduli, the traditional flavor symmetry of the $\mathbb{T}^{2} / \mathbb{Z}_{2}$ orbifold is $\left(D_{8} \times D_{8}\right) / \mathbb{Z}_{2} \cong[32,49]$, cf. eq. (2.13), associated with the discrete symmetries of the theory that do not affect the moduli.

On the other hand, as explained in section 3, omitting the $\mathcal{C P}$-like transformation $\hat{\Sigma}_{*}$ in a first step, the finite modular group of the $\mathbb{T}^{2} / \mathbb{Z}_{2}$ orbifold is $\left(S_{3}^{T} \times S_{3}^{U}\right) \rtimes \mathbb{Z}_{4}^{\hat{M}} \cong[144,115]$. 
This group can be constructed by the order 4 generator $\rho_{\mathbf{4}}(\hat{M})$ associated with the mirror transformation $\hat{M}$, see eq. (3.6), and the $S_{3}^{T} \times S_{3}^{U}$ finite modular transformations arising from $\mathrm{SL}(2, \mathbb{Z})_{T}$ and $\mathrm{SL}(2, \mathbb{Z})_{U}$. These modular symmetries are, in general, independent of the traditional flavor symmetry because traditional flavor transformations act trivially on the moduli $T$ and $U$. In contrast, modular transformations act by definition nontrivially on these moduli. We assume now that the moduli are fixed at some vacuum expectation values (vevs) $(\langle T\rangle,\langle U\rangle)$, see e.g. ref. [31]. Then, we have to distinguish between two cases: first, there are modular transformations that do not leave the moduli vevs invariant. They are broken spontaneously. Second, some modular transformations may leave the moduli vevs invariant. They build the so-called stabilizer subgroup

$$
\left.H_{(\langle T\rangle,\langle U\rangle)}:=\langle\gamma| \gamma \in \Xi \quad \text { with } \quad \gamma(\langle T\rangle)=\langle T\rangle \text { and } \gamma(\langle U\rangle)=\langle U\rangle\right\rangle
$$

which depends on the moduli vevs $\langle T\rangle$ and $\langle U\rangle$. By definition, $H_{(\langle T\rangle,\langle U\rangle)}$ is a subgroup of $\Xi=\mathrm{O}_{\hat{\eta}}(2,2, \mathbb{Z}) / \mathbb{Z}_{2}$, see eq. (B.6). As detailed in appendix B, $\Xi$ is given by the full modular group $\mathrm{O}_{\hat{\eta}}(2,2, \mathbb{Z})$ defined in eq. (A.9) divided by the $\mathbb{Z}_{2}$ point group, where $\mathrm{O}_{\hat{\eta}}(2,2, \mathbb{Z})$ comprises $\operatorname{SL}(2, \mathbb{Z})_{T}, \operatorname{SL}(2, \mathbb{Z})_{U}$, the mirror transformation $\hat{M}$, and the $\mathcal{C} \mathcal{P}$-like transformation $\hat{\Sigma}_{*}$. If the stabilizer subgroup at a special point in moduli space is nontrivial, i.e. $H_{(\langle T\rangle,\langle U\rangle)} \neq\{\mathbb{1}\}$, then its elements remain unbroken by the vevs. Due to their trivial action on the moduli vevs and nontrivial action on matter fields, these unbroken transformations enhance the traditional flavor group to a so-called unified flavor group

$$
\frac{D_{8} \times D_{8}}{\mathbb{Z}_{2}} \cup H_{(\langle T\rangle,\langle U\rangle)},
$$

which results from the multiplicative closure of the universal traditional flavor group and the stabilizer subgroup $H_{(\langle T\rangle,\langle U\rangle)}$.

Thus, given the representation of the elements of the traditional flavor group in the field basis, determining the unified flavor group at the point $(\langle T\rangle,\langle U\rangle)$ requires to know the matrix representation $\rho(\gamma)$ associated with the action of the element $\gamma$ of the stabilizer subgroup $H_{(\langle T\rangle,\langle U\rangle)}$ on the orbifold matter fields $\Phi$. For the four twisted matter fields of the $\mathbb{T}^{2} / \mathbb{Z}_{2}$ orbifold, $\rho(\gamma)$ can be built from the $\rho_{4}$ representation matrices given in eqs. (3.5), (3.6) and (3.8).

However, since string matter fields carry (fractional) modular weights $\left(n_{T}, n_{U}\right)$ of $\mathrm{SL}(2, \mathbb{Z})_{T}$ and $\mathrm{SL}(2, \mathbb{Z})_{U}$, the matrix representation $\rho(\gamma)$ of a modular transformation $\gamma$ at the point $(\langle T\rangle,\langle U\rangle)$ in moduli space is accompanied by so-called automorphy factors of the form $\left(c_{T}\langle T\rangle+d_{T}\right)^{n_{T}}\left(c_{U}\langle U\rangle+d_{U}\right)^{n_{U}}$, where $c_{T}, d_{T}, c_{U}, d_{U}$ are integers parametrizing the transformation $\gamma \in H_{(\langle T\rangle,\langle U\rangle)}$. As discussed in ref. [12], these automorphy factors evaluated at the vevs $(\langle T\rangle,\langle U\rangle)$ are discrete phases. This fact can i) change the order of the flavor symmetry associated with $\gamma$, and/or ii) reveal that $\gamma$ acts as a discrete $R$-symmetry, which in general promotes the unified flavor group at $(\langle T\rangle,\langle U\rangle)$ to a non-Abelian discrete $R$-symmetry of $\mathcal{N}=1$ supersymmetry [32]. However, for the sake of clarity and simplicity, we ignore the automorphy factors in the following. Hence, hereafter we shall provide the actual groups of the unified flavor symmetries only in cases where the automorphy factors 
do not affect the results, but shall give in all cases the generators of the corresponding stabilizer subgroups. The subtleties about the consequences of the automorphy factors as well as their relevance in six-dimensional orbifolds shall be discussed in detail later in ref. [33].

A first example of a unified flavor symmetry arises at $\langle T\rangle=\langle U\rangle$ with generic vev. This is a two-dimensional hypersurface in four-dimensional (real) moduli space. At these points, the mirror symmetry transformation $\hat{M}$, acting on the moduli as $T \stackrel{\hat{M}}{\longleftrightarrow} U$, generates a $\mathbb{Z}_{2}$ stabilizer subgroup. Considering the representation $\rho_{\mathbf{4}}(\hat{M})$ of $\hat{M}$ for twisted matter fields, eq. (3.6), one finds that the traditional flavor group

$$
[32,49] \cong\left(D_{8} \times D_{8}\right) / \mathbb{Z}_{2} \quad \text { enhances to } \quad[64,257] \text { at } \quad\langle T\rangle=\langle U\rangle .
$$

Note that, although the order of $\rho_{\mathbf{4}}(\hat{M})$ is 4 , the order of the flavor group is enhanced only by a factor of 2 because $\left(\rho_{4}(\hat{M})\right)^{2}=-\mathbb{1}_{4}$ is also included in the traditional flavor group, cf. eq. (3.7).

Mirror symmetry $\hat{M}$ helps to simplify the study of unified flavor symmetries at special points in moduli space. We know that the mirror transformation $\hat{M}$ maps

$$
U \stackrel{\hat{M}}{\longleftrightarrow} T, \quad \hat{K}_{\mathrm{S}} \stackrel{\hat{M}}{\longleftrightarrow} \hat{C}_{\mathrm{S}} \quad \text { and } \quad \hat{K}_{\mathrm{T}} \stackrel{\hat{M}}{\longleftrightarrow} \hat{C}_{\mathrm{T}}
$$

cf. eq. (A.13) in appendix A.2. Since $\hat{M}$ is a symmetry of the theory, there is an equivalence between the unbroken modular symmetry at the point $(T, U)=(\langle T\rangle,\langle U\rangle)$ in moduli space and its mirror dual at the point $(T, U)=(\langle U\rangle,\langle T\rangle)$. This implies that the stabilizer subgroups satisfy the isomorphism

$$
H_{(T=\langle T\rangle, U=\langle U\rangle)} \cong H_{(T=\langle U\rangle, U=\langle T\rangle)},
$$

using $\hat{M} \gamma \hat{M}^{-1} \in H_{(T=\langle U\rangle, U=\langle T\rangle)}$ for all $\gamma \in H_{(T=\langle T\rangle, U=\langle U\rangle)}$ and $\hat{M} h \hat{M}^{-1} \in\left(D_{8} \times D_{8}\right) / \mathbb{Z}_{2}$ for all $h \in\left(D_{8} \times D_{8}\right) / \mathbb{Z}_{2}$. Consequently, the associated unified flavor symmetries, resulting from combining these stabilizer subgroups with the universal traditional flavor group, as prescribed by eq. (4.2), are isomorphic too. This implies that mirror symmetry $\hat{M}$ halves the fundamental domain in the full moduli space and we must not explore both isomorphic cases eq. (4.5) independently. Thus, we shall explore only those symmetry enhanced points and hypersurfaces in moduli space that are associated with a geometric interpretation of $U$. Then, for each case of $\langle U\rangle$, we identify the symmetry enhanced points in the $T$-moduli space. Hence, we can restrict ourselves to four special cases:

i) the generic $\mathbb{T}^{2} / \mathbb{Z}_{2}$ orbifold with generic vev $\langle U\rangle$,

ii) the tetrahedron with $\langle U\rangle=e^{\frac{\pi i}{3}}$,

iii) the raviolo with $\langle U\rangle=\mathrm{i}$, and

iv) $\mathbb{T}^{2} / \mathbb{Z}_{2}$ orbifolds with $\mathcal{C} \mathcal{P}$-enhancement.

In the following, we will discuss these four cases in detail, restricting to points of the fundamental domain in moduli space. 


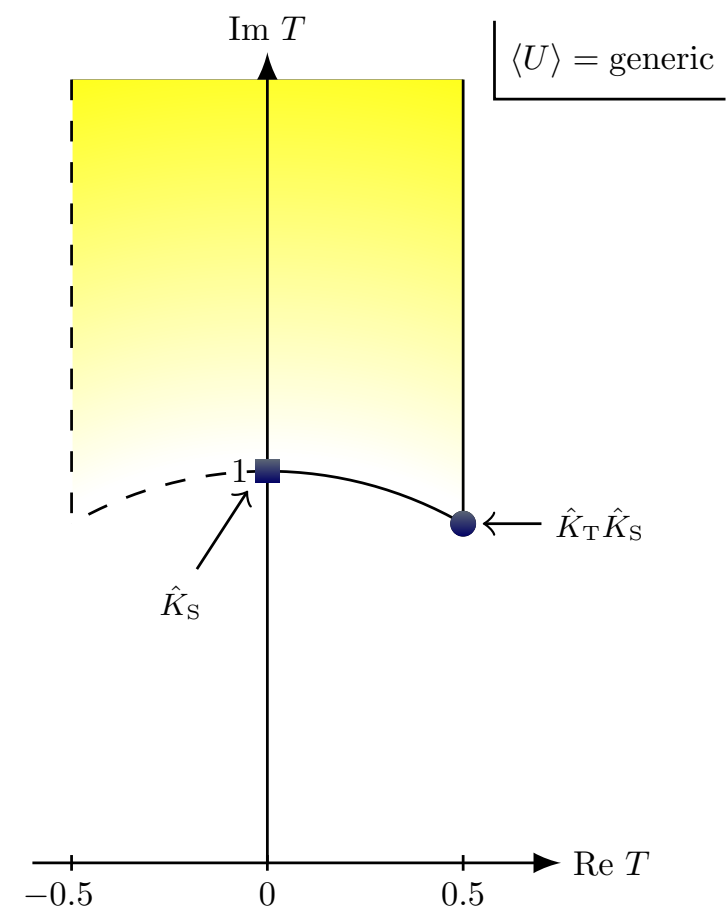

(a) $U$ fixed at a generic value $\langle U\rangle \neq \mathrm{i}, e^{\frac{\pi \mathrm{i}}{3}}$.

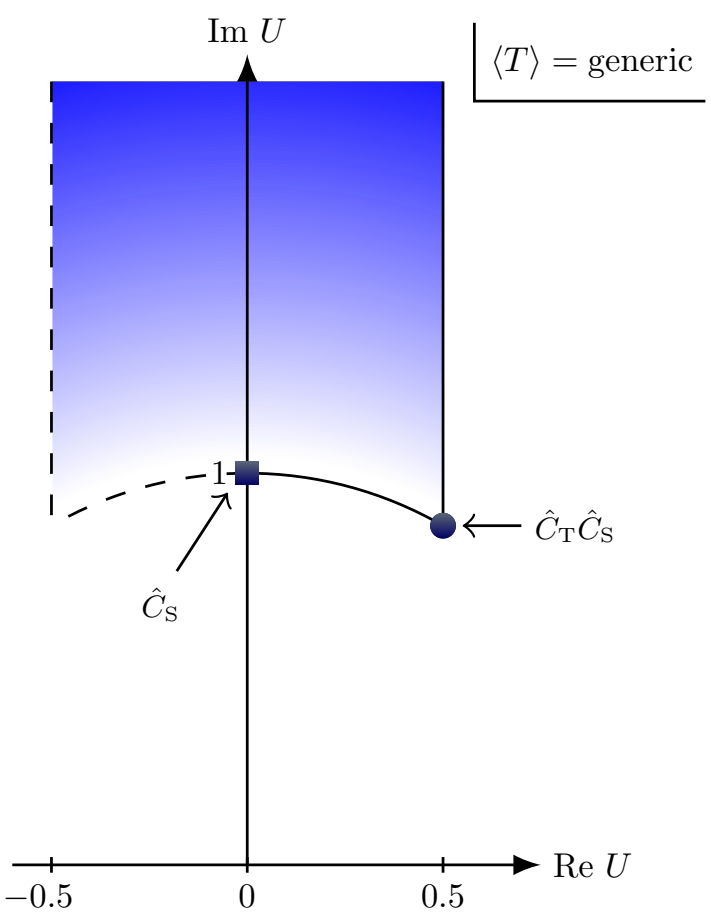

(b) $T$ fixed at a generic value $\langle T\rangle \neq \mathrm{i}, e^{\frac{\pi i}{3}}$.

Figure 4. Generators of nontrivial stabilizer subgroups at special points in moduli space. (a) For generic $\langle U\rangle$, only at $\langle T\rangle=\mathrm{i}$ (square) and $\langle T\rangle=e^{\frac{\pi \mathrm{i}}{3}}$ (bullet) the stabilizer subgroup is nontrivial. The corresponding stabilizer subgroups are $H_{(\mathrm{i},\langle U\rangle)}=\left\langle\hat{K}_{\mathrm{S}}\right\rangle \cong \mathbb{Z}_{2}$ and $H_{\left(e^{\frac{\pi \mathrm{i}}{3}},\langle U\rangle\right)}=\left\langle\hat{K}_{\mathrm{T}} \hat{K}_{\mathrm{S}}\right\rangle \cong \mathbb{Z}_{3}$. (b) For generic $\langle T\rangle$, the results are equivalent due to mirror symmetry $\hat{M}$, which exchanges $T \leftrightarrow U$, $\hat{K}_{\mathrm{T}} \leftrightarrow \hat{C}_{\mathrm{T}}$ and $\hat{K}_{\mathrm{S}} \leftrightarrow \hat{C}_{\mathrm{S}}$

\subsection{The generic $\mathbb{T}^{2} / \mathbb{Z}_{2}$ orbifold}

Let us consider first the case of a generic vev $\langle U\rangle$ of the complex structure modulus $U$. In this case, $\mathcal{C P}$ is broken by the generic vev of $U$. Furthermore, as shown in figure 4a, there are only two inequivalent special values of the Kähler modulus $T$ associated with a nontrivial stabilizer modular subgroup: $\langle T\rangle=\mathrm{i}$ and $\langle T\rangle=e^{\frac{\pi \mathrm{i}}{3}}$. At these points, we see that the Kähler modulus is invariant under the transformations

$$
\begin{array}{llrl}
\text { at }\langle T\rangle=\mathrm{i} & : & \langle T\rangle \stackrel{\hat{K}_{\mathrm{S}}}{\longrightarrow}-\frac{1}{\langle T\rangle}=\langle T\rangle, \\
\text { at }\langle T\rangle=e^{\frac{\pi \mathrm{i}}{3}} & : & \langle T\rangle \stackrel{\hat{K}_{\mathrm{T}}}{\longrightarrow}\langle T\rangle+1 \stackrel{\hat{K}_{\mathrm{S}}}{\longrightarrow}-\frac{1}{\langle T\rangle}+1=\langle T\rangle,
\end{array}
$$

and $\langle U\rangle$ is not affected, cf. eq. (3.1). The corresponding stabilizer subgroups are

$$
\begin{aligned}
& \text { at }\langle T\rangle=\mathrm{i} \quad \text { : } \quad H_{(\mathrm{i},\langle U\rangle)}=\left\langle\hat{K}_{\mathrm{S}} \mid\left(\hat{K}_{\mathrm{S}}\right)^{2} \sim \mathbb{1}\right\rangle \cong \mathbb{Z}_{2}, \\
& \text { at }\langle T\rangle=e^{\frac{\pi \mathrm{i}}{3}} \quad: \quad H_{\left(e^{\frac{\pi \mathrm{i}}{3}},\langle U\rangle\right)}=\left\langle\hat{K}_{\mathrm{T}} \hat{K}_{\mathrm{S}} \mid\left(\hat{K}_{\mathrm{T}} \hat{K}_{\mathrm{S}}\right)^{3}=\mathbb{1}\right\rangle \cong \mathbb{Z}_{3} \text {. }
\end{aligned}
$$


Note that $\left(\hat{K}_{\mathrm{S}}\right)^{2}$ is a trivial element of all stabilizer subgroups because the stabilizer subgroup is defined up to point group transformations (see the discussion around eq. (4.1)) and $\left(\hat{K}_{\mathrm{S}}\right)^{2}=\left(\hat{C}_{\mathrm{S}}\right)^{2}=-\mathbb{1}_{4}$ is equivalent to the $\mathbb{Z}_{2}$ point group generator $\hat{\Theta}=-\mathbb{1}_{4}$. It is easy to confirm the mirror duals of eqs. (4.7), as shown in figure $4 \mathrm{~b}$ for generic $\langle T\rangle$.

To exemplify the enhancement of the traditional flavor symmetry to unified flavor groups, let us focus on the hypersurface $\langle T\rangle=e^{\frac{\pi \mathrm{i}}{3}}$ for generic $\langle U\rangle$. We use the $\rho_{\mathbf{4}}$ representation of $\hat{K}_{\mathrm{T}}$ and $\hat{K}_{\mathrm{S}}$ given in eqs. (3.8), where $\left(\rho_{\mathbf{4}}\left(\hat{K}_{\mathrm{S}}\right)\right)^{2}=\mathbb{1}_{4}$, together with those of the generators of the traditional flavor group, eqs. (2.11) and eqs. (2.12). In this way, we find that the nontrivial stabilizer subgroup enhances

$$
\left(D_{8} \times D_{8}\right) / \mathbb{Z}_{2} \quad \text { to }[96,204] \quad \text { at }\langle U\rangle=\text { generic, }\langle T\rangle=e^{\frac{\pi \mathrm{i}}{3}} .
$$

As intuitively expected, the order is 3 times as large as the order of the original group $\left(D_{8} \times D_{8}\right) / \mathbb{Z}_{2} \cong[32,49]$ because of the nontrivial $\mathbb{Z}_{3}$ factor introduced by the stabilizer. Due to mirror symmetry, this unified flavor symmetry is isomorphic to the resulting unified flavor symmetry at $\langle U\rangle=e^{\frac{\pi \mathrm{i}}{3}}$ for generic $\langle T\rangle$.

After this first case, we can now proceed to study the more complex cases of the $\mathbb{T}^{2} / \mathbb{Z}_{2}$ orbifold adopting the shapes of a tetrahedron and a raviolo, where the $\mathcal{C P}$-like modular transformation $\hat{\Sigma}_{*}$ plays an important role.

\subsection{The tetrahedron with $\langle U\rangle=e^{\frac{\pi \mathrm{i}}{3}}$}

Setting $\langle U\rangle=e^{\frac{\pi \mathrm{i}}{3}}$ leads to the $\mathbb{T}^{2} / \mathbb{Z}_{2}$ illustrated in figure 5 . On the left, we see the corresponding two-dimensional torus lattice spanned by $e_{1}$ and $e_{2}$ of equal length and enclosing an angle of $\frac{\pi}{3}$. Modding out a $\mathbb{Z}_{2}$ symmetry of the two-torus reduces the fundamental domain to the yellow region, whose boundaries are identified as the arrows indicate. These identifications allow for the fundamental domain of the $\mathbb{T}^{2} / \mathbb{Z}_{2}$ orbifold to adopt the shape of the tetrahedron displayed on the right of the figure. The corners of the tetrahedron correspond to the four fixed points of the orbifold, where the twisted matter fields $\left(\phi_{(0,0)}, \phi_{(1,0)}, \phi_{(0,1)}, \phi_{(1,1)}\right)^{\mathrm{T}}$ are localized.

The generic flavor symmetry group of the tetrahedron can be found by considering the stabilizer subgroup at $\langle U\rangle=e^{\frac{\pi \mathrm{i}}{3}}$ for generic $\langle T\rangle$,

$$
H_{\left(\langle T\rangle, e^{\frac{\pi \mathrm{i}}{3}}\right)}=\left\langle\hat{C}_{\mathrm{T}} \hat{C}_{\mathrm{S}}\right\rangle \cong \mathbb{Z}_{3},
$$

which corresponds to the mirror dual of eq. (4.7b). In this case, the unified flavor group is constructed by the generators of the traditional flavor group and $\hat{C}_{\mathrm{T}} \hat{C}_{\mathrm{S}}$. The resulting unified flavor group is [96, 204], as in the dual scenario given in eq. (4.8).

Since the stabilizer generator $\hat{C}_{\mathrm{T}} \hat{C}_{\mathrm{S}}$ is now a symmetry everywhere in Kähler moduli space, it is displayed in the fundamental domain (yellow area) of figure 6a. If we now consider the $\mathcal{C} \mathcal{P}$-like transformation $\hat{\Sigma}_{*}$, for particular values of $\langle T\rangle$, the flavor group of the tetrahedron is enhanced further. These enhancements occur at the points $\langle T\rangle$ along the curve $\lambda_{T}$ of figure $6 \mathrm{a}$, which is the boundary of the fundamental domain in $T$ moduli space. The orientation of the curve $\lambda_{T}$ is indicated by bold arrows. It will be used later in section 4.4. 

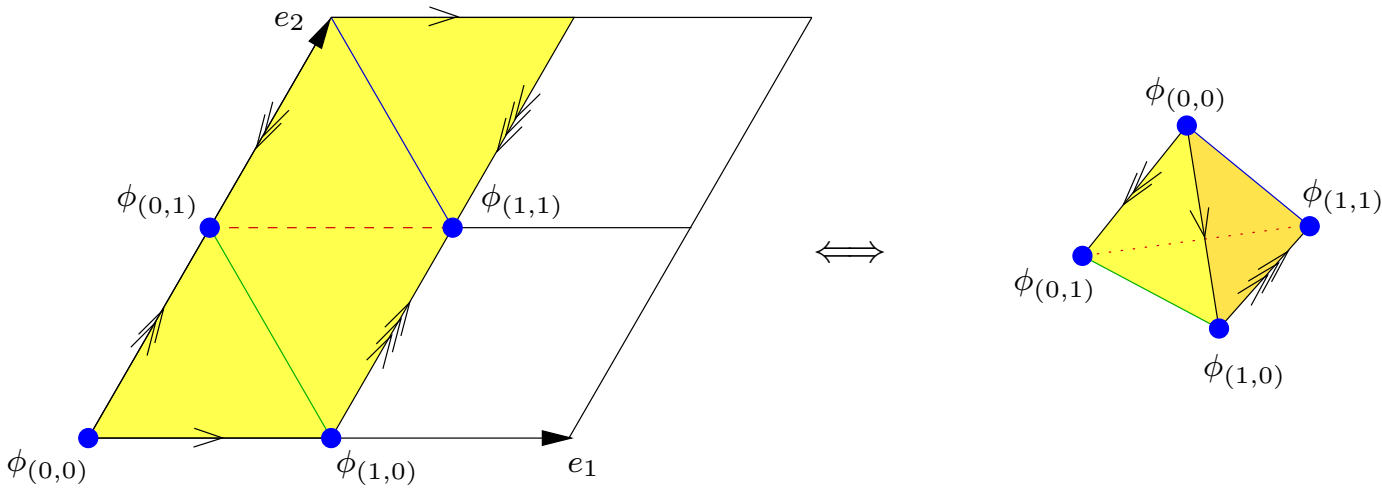

Figure 5. The $\mathbb{T}^{2} / \mathbb{Z}_{2}$ orbifold with $\langle U\rangle=e^{\frac{\pi i}{3}}$. Identifying the arrows indicated on the boundaries of the fundamental domain of the orbifold yields a tetrahedron, whose vertex corners correspond to the four fixed points of the orbifold, where twisted matter fields $\phi_{\left(n_{1}, n_{2}\right)}$ are localized.

For example, at the points of the curve $\lambda_{T}$ with $\operatorname{Re}\langle T\rangle=\frac{1}{2}$ and $\operatorname{Im}\langle T\rangle>\frac{\sqrt{3}}{2}$ we find the $\mathcal{C P}$-like transformation $\hat{K}_{\mathrm{T}} \hat{C}_{\mathrm{S}} \hat{\Sigma}_{*}$. It acts on the moduli as

$$
\begin{array}{r}
\langle T\rangle \stackrel{\hat{K}_{\mathrm{T}}}{\longrightarrow}\langle T\rangle+1 \stackrel{\hat{C}_{\mathrm{S}}}{\longrightarrow}\langle T\rangle+1 \stackrel{\hat{\Sigma}_{*}}{\longrightarrow}-\langle\bar{T}\rangle+1=\langle T\rangle, \\
\langle U\rangle \stackrel{\hat{K}_{\mathrm{T}}}{\longrightarrow}\langle U\rangle \stackrel{\hat{C}_{\mathrm{S}}}{\longrightarrow}-\frac{1}{\langle U\rangle} \stackrel{\hat{\Sigma}_{*}}{\longrightarrow} \frac{1}{\langle\bar{U}\rangle}=\langle U\rangle .
\end{array}
$$

Hence, it belongs to the stabilizer subgroup

$$
H_{\left(\frac{1}{2}+\mathrm{i} \operatorname{Im}\langle T\rangle, e^{\frac{\pi \mathrm{i}}{3}}\right)}=\left\langle\hat{C}_{\mathrm{T}} \hat{C}_{\mathrm{S}}, \hat{K}_{\mathrm{T}} \hat{C}_{\mathrm{S}} \hat{\Sigma}_{*}\right\rangle, \quad \text { with } \quad \operatorname{Im}\langle T\rangle>\frac{\sqrt{3}}{2} .
$$

Using the representations of the involved modular transformations, given in eqs. (3.5), (3.8) and (3.9), leads to the unified flavor group [192,1494], which is known as $S W_{4}$ and can be associated with all pure rotations of a four-dimensional cube [34]. In contrast to ref. [13], we realize the $S W_{4}$ symmetry only if both moduli $T$ and $U$ take special values. The same enhancement results for $\langle T\rangle=e^{\mathrm{i} \varphi}$ with $\frac{\pi}{3}<\varphi<\frac{\pi}{2}$, where, as indicated in figure 6a, the stabilizer subgroup $H_{\left(e^{\mathrm{i} \varphi}, e^{\frac{\pi \mathrm{i}}{3}}\right)}$ is generated by $\hat{C}_{\mathrm{T}} \hat{C}_{\mathrm{S}}$ and $\hat{K}_{\mathrm{S}} \hat{C}_{\mathrm{S}} \hat{\Sigma}_{*}$.

Another interesting example is the maximally symmetric point $\langle T\rangle=\langle U\rangle=e^{\frac{\pi \mathrm{i}}{3}}$. There, in addition to $\hat{C}_{\mathrm{T}} \hat{C}_{\mathrm{S}}$, also the mirror transformation $\hat{M}$ and the $\mathcal{C P}$-like transformation

$$
\begin{array}{r}
\langle T\rangle \stackrel{\hat{C}_{\mathrm{T}}}{\longrightarrow}\langle T\rangle \stackrel{\hat{K}_{\mathrm{T}}}{\longrightarrow}\langle T\rangle+1 \stackrel{\hat{\Sigma}_{*}}{\longrightarrow}-\langle\bar{T}\rangle+1=\langle T\rangle, \\
\langle U\rangle \stackrel{\hat{C}_{\mathrm{T}}}{\longrightarrow}\langle U\rangle+1 \stackrel{\hat{K}_{\mathrm{T}}}{\longrightarrow}\langle U\rangle+1 \stackrel{\hat{\Sigma}_{*}}{\longrightarrow}-\langle\bar{U}\rangle+1=\langle U\rangle
\end{array}
$$

build the stabilizer subgroup

$$
H_{\left(e^{\frac{\pi \mathrm{i}}{3}}, e^{\frac{\pi \mathrm{i}}{3}}\right)}=\left\langle\hat{C}_{\mathrm{T}} \hat{C}_{\mathrm{S}}, \hat{C}_{\mathrm{T}} \hat{K}_{\mathrm{T}} \hat{\Sigma}_{*}, \hat{M}\right\rangle,
$$

as displayed in figure 6a. Using the representations of these generators acting on twisted matter fields, including eq. (3.6), we find that the generic flavor symmetry of the tetrahedron $[96,204]$ is enhanced to the unified flavor group [1152,157463]. This corresponds 


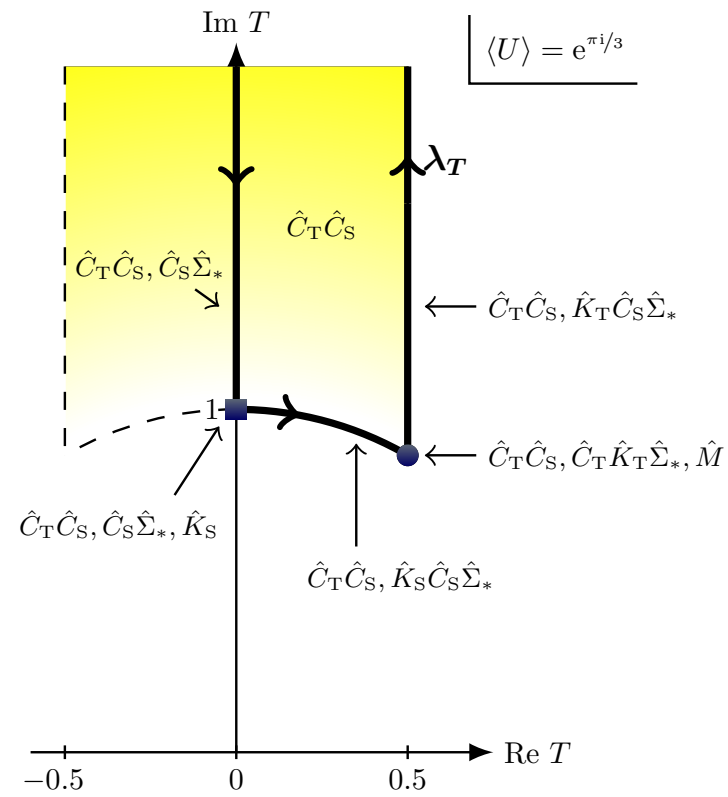

(a) $\mathbb{T}^{2} / \mathbb{Z}_{2}$ with $\langle U\rangle=e^{\frac{\pi \mathrm{i}}{3}}$.

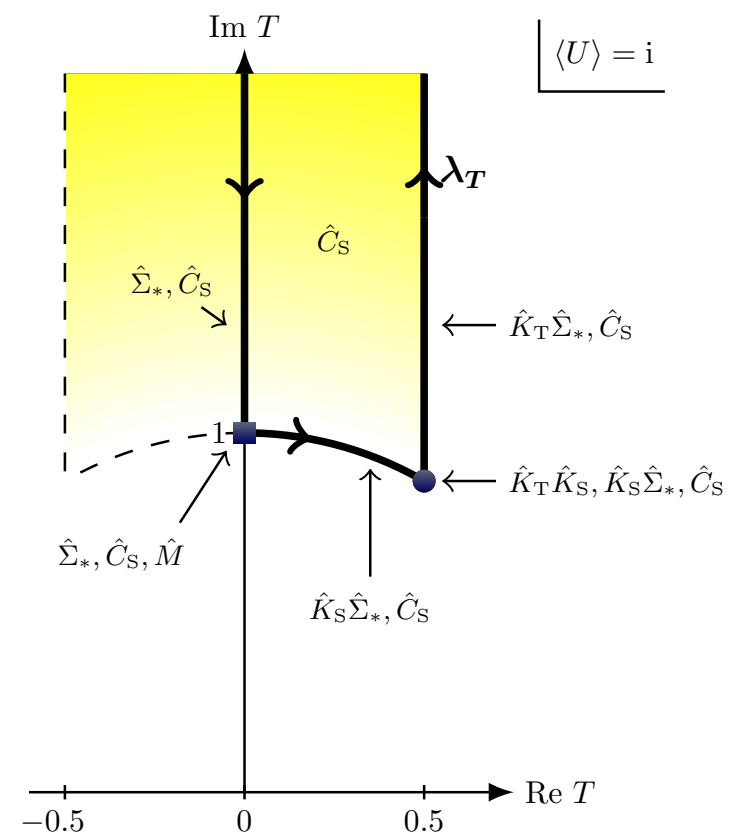

(b) $\mathbb{T}^{2} / \mathbb{Z}_{2}$ with $\langle U\rangle=$ i.

Figure 6. Generators of the stabilizer subgroups $H_{(\langle T\rangle,\langle U\rangle)}$ at different points $\langle T\rangle$ of the fundamental domain of $\mathrm{SL}(2, \mathbb{Z})_{T}$ for two special vevs $\langle U\rangle$ of the complex structure modulus. These elements enhance the traditional flavor symmetry to various unified flavor groups.

to the largest enhancement of the traditional flavor group in the $\mathbb{T}^{2} / \mathbb{Z}_{2}$ orbifold, including mirror symmetry and $\mathcal{C P}$. We observe that even in the simplest case of a $\mathbb{T}^{2} / \mathbb{Z}_{2}$ orbifold, the flavor symmetry can be very large.

Other nontrivial stabilizer subgroup generators are displayed along the curve $\lambda_{T}$ of figure 6a.

\subsection{The raviolo with $\langle U\rangle=\mathrm{i}$}

Fixing the complex structure modulus to $\langle U\rangle=\mathrm{i}$ amounts to setting $\left|e_{1}\right|=\left|e_{2}\right|$ with an angle of $\frac{\pi}{2}$ between them. This corresponds to a toroidal lattice whose basis vectors are orthogonal and have equal length. In this case, the $\mathbb{T}^{2} / \mathbb{Z}_{2}$ orbifold takes the shape of a raviolo, similar to the one depicted in figure 1, but whose edges are perpendicular and all have the same length.

The stabilizer subgroup for generic $\langle T\rangle$ and $\langle U\rangle=\mathrm{i}$ is dual to the one given in eq. (4.7a): it is generated by $\hat{C}_{\mathrm{S}}$. Note that at $\langle U\rangle=\mathrm{i}$ the transformation $\hat{C}_{\mathrm{S}}$ gives rise to a $\frac{\pi}{2}$ rotation in the compact dimensions. Hence, $\hat{C}_{\mathrm{S}}$ is a discrete remnant of the higher-dimensional Lorentz symmetry. Thus, it generates an $R$-symmetry. In figure $6 \mathrm{~b}$, this universal generator of the stabilizer subgroups is displayed in the fundamental domain in the $T$ moduli space (yellow area). Similarly to the previous case, taking into account the $\mathcal{C} \mathcal{P}$-like transformation $\hat{\Sigma}_{*}$, further enhancements arise from the elements of the stabilizer subgroups shown in figure $6 \mathrm{~b}$ at the points $\langle T\rangle$ along the curve $\lambda_{T}$. 


\section{$4.4 \quad \mathbb{T}^{2} / \mathbb{Z}_{2}$ orbifolds with $\mathcal{C} \mathcal{P}$-enhancement}

In figure 7 , we display the landscape of all nontrivial stabilizers that contain $\mathcal{C P}$-like generators. To do so, we use the straightened curves $\lambda_{T}$ and $\lambda_{U}$ as the axes in figure 7 . To be specific, the curve $\lambda_{T}$ is defined as the boundary of the fundamental domain of the $S_{3}^{T}$ modular symmetry in figure 6 , where the orientation is illustrated by the bold arrows. Its mirror dual $\lambda_{U}$ is defined analogously in the $U$-modulus plane. Hence, the horizontal $\lambda_{T}$ axis must be interpreted as follows:

- between the leftmost point and $\lambda_{T}=\mathrm{i}, \lambda_{T}$ corresponds to $\infty>\operatorname{Im}\langle T\rangle \geq 1$ and $\operatorname{Re}\langle T\rangle=0$,

- for $\lambda_{T}$ between $\mathrm{i}$ and $e^{\frac{\pi \mathrm{i}}{3}}$, the curve $\lambda_{T}$ describes $\langle T\rangle=e^{\mathrm{i} \varphi}$ with $\frac{\pi}{2}>\varphi>\frac{\pi}{3}$, and

- between $\lambda_{T}=e^{\frac{\pi \mathrm{i}}{3}}$ and the rightmost point, the curve $\lambda_{T}$ is associated with $\frac{\sqrt{3}}{2} \leq$ $\operatorname{Im}\langle T\rangle\left\langle\infty\right.$ and $\operatorname{Re}\langle T\rangle=\frac{1}{2}$.

Similarly, the dual vertical axis $\lambda_{U}$ has to be read bottom-up, exchanging $\langle T\rangle$ in the previous description by $\langle U\rangle$. The color schema is such that points with values closer to $\langle T\rangle \rightarrow \mathrm{i} \infty(\langle U\rangle \rightarrow \mathrm{i} \infty)$ are more yellow (blue). Large imaginary values of both $\langle T\rangle$ and $\langle U\rangle$ yield the green texture.

We observe that the results presented in figures $6 \mathrm{a}$ and $6 \mathrm{~b}$ are reproduced in figure 7 along the upper and lower horizontal lines, respectively. Mirror symmetry maps the lower (upper) horizontal line to the left (right) vertical line, where the stabilizer generators coincide after performing the transformations $\hat{C}_{\mathrm{S}} \stackrel{\hat{M}}{\longleftrightarrow} \hat{K}_{\mathrm{S}}$ and $\hat{C}_{\mathrm{T}} \stackrel{\hat{M}}{\longleftrightarrow} \hat{K}_{\mathrm{T}}$.

The diagonal in figure 7 describes all special points in moduli space at which $\langle T\rangle=\langle U\rangle$ is satisfied. We note that there are different enhancements depending on the particular values of the moduli. For example, for $\langle T\rangle=\langle U\rangle=\mathrm{i} x$ with $x>1$, the stabilizer subgroup is $H_{(\mathrm{i} x, \mathrm{i} x)}=\left\langle\hat{\Sigma}_{*}, \hat{M}\right\rangle$, as displayed on the lower left panel of the figure. As a final example, consider the area $\lambda_{T}<\mathrm{i}$ and $\mathrm{i}<\lambda_{U}<e^{\frac{\pi \mathrm{i}}{3}}$ in figure 7, i.e. the middle left panel (equivalently defined by $\langle T\rangle=\mathrm{i} x$ with $x>1$ and $\langle U\rangle=e^{\mathrm{i} \varphi}$ for $\frac{\pi}{2}>\varphi>\frac{\pi}{3}$ ). In this case, the stabilizer subgroup is generated by the $\mathcal{C} \mathcal{P}$-like transformation $\hat{C}_{\mathrm{S}} \hat{\Sigma}_{*}$. In summary, we obtain all nontrivial stabilizers that involve $\mathcal{C P}$-enhancement and illustrate them in figure 7 .

\section{Conclusions and outlook}

We have seen that modular transformations of the $\mathbb{T}^{2} / \mathbb{Z}_{2}$ orbifold lead to an exceedingly rich eclectic structure. From the traditional flavor symmetry $D_{8} \times D_{8} / \mathbb{Z}_{2} \cong[32,49]$, we obtain an eclectic flavor group with as many as 4608 elements. We also observe large flavor groups that are linearly realized at specific regions in $T$-and $U$-moduli space, the largest being $[1152,157463]$ at $\langle T\rangle=\langle U\rangle=\exp \left(\frac{\pi \mathrm{i}}{3}\right)$. For reasons of clarity and simplicity, the discussion in the present paper has concentrated on group theoretical studies of finite modular groups and we have not yet included a full implementation of the automorphy factors of $\operatorname{SL}(2, \mathbb{Z})_{T}$ and $\operatorname{SL}(2, \mathbb{Z})_{U}$. Future work [33] will include this along the lines explained in refs. $[12,16]$ for the $\mathbb{T}^{2} / \mathbb{Z}_{3}$ orbifold. As discussed in ref. [12], the automorphy 


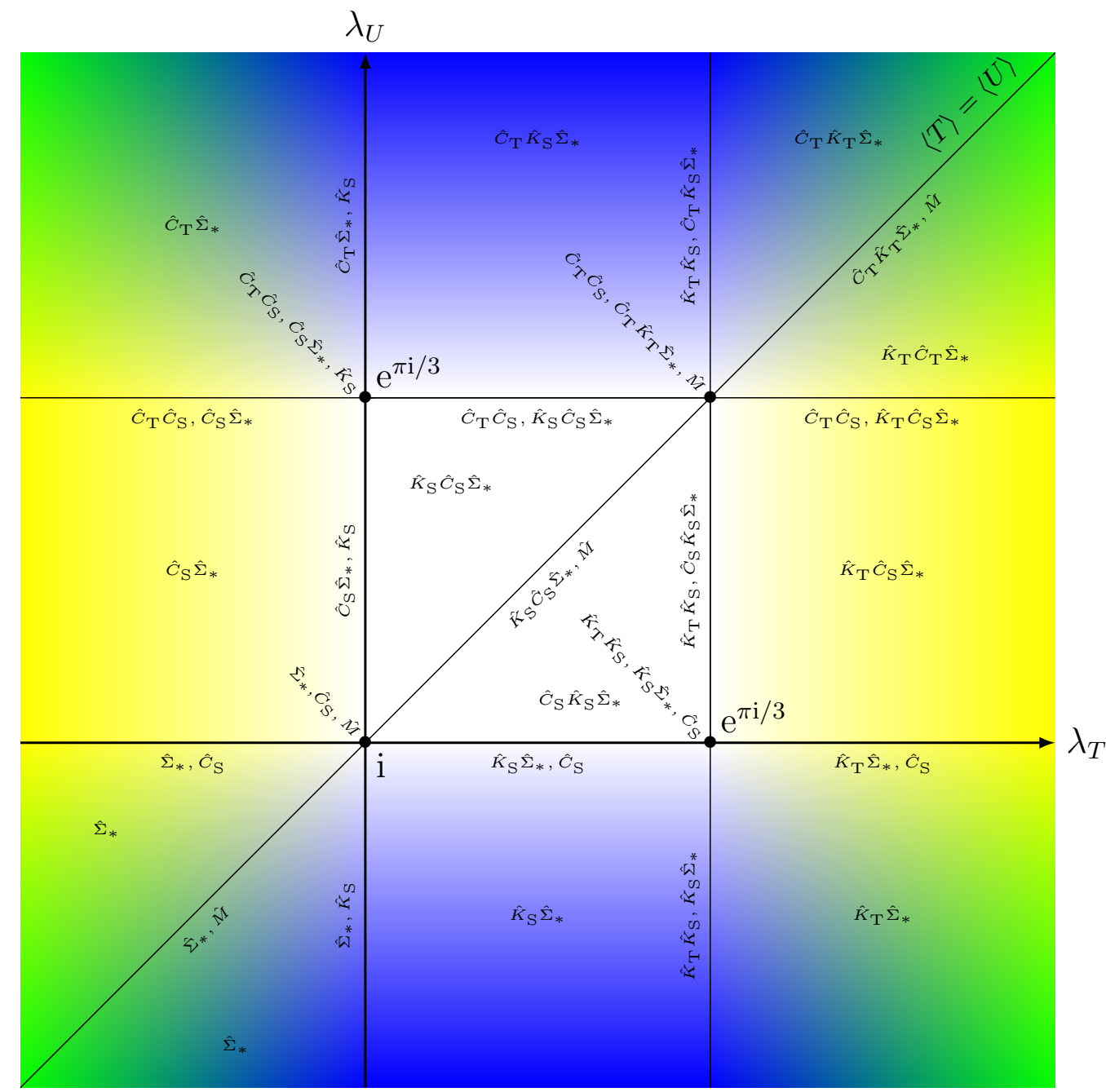

Figure 7. Generators of the stabilizers $H_{(\langle T\rangle,\langle U\rangle)}$ for $\langle T\rangle \in \lambda_{T}$ and $\langle U\rangle \in \lambda_{U}$. The axes $\lambda_{T}$ and $\lambda_{U}$ correspond to the curves on the boundaries of the two-dimensional fundamental domains of $T$ and $U$, see e.g. figure 6 for $\lambda_{T}$. The diagonal depicts the hypersurface where $\langle U\rangle=\langle T\rangle$ on the curves $\lambda_{T}$ and $\lambda_{U}$. The stabilizers above and below the diagonal are related by mirror symmetry $\hat{M}$.

factors lead to discrete phases. These give rise to discrete $R$-symmetries that complete the full eclectic structure. Together with the results presented in this paper, this will be a crucial step towards a discussion of orbifolds with six compact extra dimensions. The general case in $D=6$ will be too difficult to be analyzed in detail. Using our $D=2$ building blocks we can simplify the discussion and consider elliptic fibrations of the $D=6$ case with various $D=2$ sublattice rotations. In a further step we would then have to consider specific string models that could successfully describe the flavor structure of quarks and leptons and make contact with available botton-up constructions [7]. The predictions of the model will crucially depend on the value of the moduli that potentially break flavor and $\mathcal{C P}$ in a desirable way. Thus, the ultimate step in realistic model building will be a discussion of moduli stabilization. We think it is premature to discuss this mechanism at the moment, as the process of moduli stabilization will crucially depend on the specific models under 
consideration. So we rather first would like to construct models that have a chance for a realistic description of flavor for some values of the moduli and relegate the discussion of moduli stabilization to future work.

\section{Acknowledgments}

We thank Michael Ratz for useful discussions. A.B., S.R.-S. and P.V. are supported by the Deutsche Forschungsgemeinschaft (SFB1258). The work of S.R.-S. was partly supported by CONACyT grants F-252167 and 278017.

\section{A Narain lattice}

In this appendix, we give a brief discussion on the Narain space group of our $\mathbb{T}^{2} / \mathbb{Z}_{2}$ orbifold, its outer automorphisms and their actions on the twisted matter fields that are localized at the fixed points of the $\mathbb{T}^{2} / \mathbb{Z}_{2}$ orbifold.

\section{A.1 Orbifolds defined by the Narain space group}

The string $y(\tau, \sigma)$ can be split into right- and left-moving degrees of freedom $y_{\mathrm{R}}$ and $y_{\mathrm{L}}$, respectively, where

$$
\left(\begin{array}{l}
y \\
\tilde{y}
\end{array}\right)=\frac{1}{\sqrt{2}}\left(\begin{array}{cc}
\mathbb{1}_{2} & \mathbb{1}_{2} \\
-\mathbb{1}_{2} & \mathbb{1}_{2}
\end{array}\right)\left(\begin{array}{l}
y_{\mathrm{R}} \\
y_{\mathrm{L}}
\end{array}\right),
$$

and $\tilde{y}$ denotes the so-called dual string coordinate. Then, one defines Narain coordinates $Y$ and compactifies them on a Narain torus, i.e.

$$
Y:=\left(\begin{array}{c}
y_{\mathrm{R}} \\
y_{\mathrm{L}}
\end{array}\right) \quad \text { where } \quad Y \sim Y+E N \quad \text { and } \quad E N \in \Gamma,
$$

Here, the integer vector $N=\left(n_{1}, n_{2}, m_{1}, m_{2}\right)^{\mathrm{T}} \in \mathbb{Z}^{4}$ gives the winding numbers $\left(n_{1}, n_{2}\right)$ and the Kaluza-Klein numbers $\left(m_{1}, m_{2}\right)$. In addition, $E$ is a $4 \times 4$ vielbein matrix, the so-called Narain vielbein (given for example in refs. [10,11], changing $B$ to $-B$ ). To ensure two-dimensional worldsheet modular invariance, the Narain vielbein has to span an even, integer, self-dual lattice with metric $\eta=\operatorname{diag}(-1,-1,1,1)$ of signature $(2,2)$ : the so-called Narain lattice $\Gamma$. In a second step, one can mod out the Narain lattice by a $\mathbb{Z}_{K}$ rotational symmetry, generated by the Narain twist

$$
\Theta=\left(\begin{array}{cc}
\theta_{\mathrm{R}} & 0 \\
0 & \theta_{\mathrm{L}}
\end{array}\right) \quad \text { where } \quad \Theta^{K}=\mathbb{1}_{4} \text { and } \quad \Theta \Gamma=\Gamma .
$$

Hence, one defines an orbifold in the Narain formulation of string theory as

$$
Y \mapsto g Y:=\Theta^{k} Y+E N \sim Y,
$$

where $g=\left(\Theta^{k}, E N\right)$ is an element of the so-called Narain space group $S_{\text {Narain }}$. The orbifold is called symmetric if the Narain twist $\Theta$ acts identically on right- and left-movers, i.e. $\theta:=\theta_{\mathrm{R}}=\theta_{\mathrm{L}}$. 


\section{A.2 Outer automorphisms of the $\mathbb{Z}_{2}$ Narain space group}

Discrete symmetries of the four-dimensional effective theory obtained from orbifold compactifications are associated with the outer automorphisms of the Narain space group $S_{\text {Narain }}$. In analogy to the definition in eq. (2.7) for the geometrical space group, an outer automorphism of $S_{\text {Narain }}$ is defined as a mapping from $S_{\text {Narain }}$ to itself. In more detail, a transformation $h=(\Sigma, E T) \notin S_{\text {Narain }}$ is an outer automorphism of $S_{\text {Narain }}$ if

$$
g \stackrel{h}{\longmapsto} h^{-1} g h \stackrel{!}{\in} S_{\text {Narain }},
$$

for all $g=\left(\Theta^{k}, E N\right) \in S_{\text {Narain }}$. In addition, $\Sigma$ has to preserve the Narain metric

$$
\Sigma^{\mathrm{T}} \eta \Sigma=\eta \text {. }
$$

Next, we translate the Narain space group and its outer automorphisms into the lattice basis, in which we denote all quantities in general by a hat. For example, eq. (A.5) reads in the lattice basis

$$
\hat{g} \stackrel{\hat{h}}{\longmapsto} \hat{h}^{-1} \hat{g} \hat{h} \stackrel{!}{\in} \hat{S}_{\text {Narain }},
$$

where

$$
\begin{aligned}
& \hat{g}:=\left(E^{-1}, 0\right)\left(\Theta^{k}, E N\right)(E, 0)=\left(\hat{\Theta}^{k}, N\right) \in \hat{S}_{\text {Narain }} \quad \text { and } \\
& \hat{h}:=\left(E^{-1}, 0\right)(\Sigma, E T)(E, 0)=(\hat{\Sigma}, T) \notin \hat{S}_{\text {Narain }} .
\end{aligned}
$$

Here, we have defined $\hat{\Theta}:=E^{-1} \Theta E$ and $\hat{\Sigma}:=E^{-1} \Sigma E$. Furthermore, due to eq. (A.6) we have to impose $\hat{\Sigma} \in \mathrm{O}_{\hat{\eta}}(2,2, \mathbb{Z})$, where the group $\mathrm{O}_{\hat{\eta}}(2,2, \mathbb{Z})$ of "rotational" outer automorphisms of the Narain lattice $\Gamma$ is defined as

$$
\left.\mathrm{O}_{\hat{\eta}}(2,2, \mathbb{Z}):=\langle\hat{\Sigma}| \hat{\Sigma} \in \mathrm{GL}(4, \mathbb{Z}) \quad \text { with } \quad \hat{\Sigma}^{\mathrm{T}} \hat{\eta} \hat{\Sigma}=\hat{\eta}\right\rangle,
$$

using the Narain metric in the lattice basis

$$
\hat{\eta}:=E^{\mathrm{T}} \eta E=\left(\begin{array}{cc}
0 & \mathbb{1}_{2} \\
\mathbb{1}_{2} & 0
\end{array}\right) .
$$

As discussed in detail in ref. [11], the group $\mathrm{O}_{\hat{\eta}}(2,2, \mathbb{Z})$ contains the generators

$$
\begin{aligned}
& \hat{K}_{\mathrm{S}}:=\left(\begin{array}{ll}
0 & \epsilon \\
\epsilon & 0
\end{array}\right) \quad \text { and } \quad \hat{K}_{\mathrm{T}}:=\left(\begin{array}{cc}
\mathbb{1}_{2} & 0 \\
\epsilon & \mathbb{1}_{2}
\end{array}\right), \quad \text { with } \quad \epsilon:=\left(\begin{array}{cc}
0 & 1 \\
-1 & 0
\end{array}\right) \text {, } \\
& \hat{C}_{\mathrm{S}}:=\left(\begin{array}{cc}
-\epsilon & 0 \\
0 & -\epsilon
\end{array}\right) \quad \text { and } \quad \hat{C}_{\mathrm{T}}:=\left(\begin{array}{cc}
\gamma & 0 \\
0 & \gamma^{-T}
\end{array}\right), \quad \text { with } \quad \gamma:=\left(\begin{array}{cc}
1 & -1 \\
0 & 1
\end{array}\right) \text {. }
\end{aligned}
$$

Note that, compared to refs. $[8,11]$ we redefined $\hat{K}_{\mathrm{S}}, \hat{K}_{\mathrm{T}}$ and $\hat{C}_{\mathrm{S}} . \hat{K}_{\mathrm{S}}$ and $\hat{K}_{\mathrm{T}}$ generate $\operatorname{SL}(2, \mathbb{Z})_{T}$ of the Kähler modulus $T$, while $\hat{C}_{\mathrm{S}}$ and $\hat{C}_{\mathrm{T}}$ generate $\mathrm{SL}(2, \mathbb{Z})_{U}$ of the complex structure modulus $U$. Moreover, the group $\mathrm{O}_{\hat{\eta}}(2,2, \mathbb{Z})$ contains two additional generators, given by

$$
\hat{\Sigma}_{*}:=\left(\begin{array}{cccc}
-1 & 0 & 0 & 0 \\
0 & 1 & 0 & 0 \\
0 & 0 & -1 & 0 \\
0 & 0 & 0 & 1
\end{array}\right) \quad \text { and } \hat{M}:=\left(\begin{array}{cccc}
0 & 0 & -1 & 0 \\
0 & 1 & 0 & 0 \\
-1 & 0 & 0 & 0 \\
0 & 0 & 0 & 1
\end{array}\right)
$$


The first generator $\hat{\Sigma}_{*}$ gives rise to a $\mathcal{C P}$-like transformation, while $\hat{M}$ generates a mirror symmetry, i.e. $T \stackrel{\hat{M}}{\longleftrightarrow} U$ and

$$
\hat{M} \hat{C}_{\mathrm{S}} \hat{M}^{-1}=\hat{K}_{\mathrm{S}} \quad \text { and } \quad \hat{M} \hat{C}_{\mathrm{T}} \hat{M}^{-1}=\hat{K}_{\mathrm{T}} .
$$

In order to identify the transformation of the moduli $T$ and $U$ under $\mathrm{O}_{\hat{\eta}}(2,2, \mathbb{Z})$, one can consider the generalized metric $\mathcal{H}$, defined by

$$
\mathcal{H}(T, U):=E^{\mathrm{T}} E .
$$

Under a modular transformation the Narain vielbein transforms as $E \mapsto E \hat{\Sigma}^{-1}$ for $\hat{\Sigma} \in$ $\mathrm{O}_{\hat{\eta}}(2,2, \mathbb{Z})$. Consequently, we obtain that the generalized metric transforms as

$$
\mathcal{H}(T, U) \stackrel{\hat{\Sigma}}{\longmapsto} \mathcal{H}\left(T^{\prime}, U^{\prime}\right)=\hat{\Sigma}^{-\mathrm{T}} \mathcal{H}(T, U) \hat{\Sigma}^{-1} .
$$

In this way, one can prove the transformations of the moduli, given in eqs. (3.1) and (3.2), under the $\mathrm{O}_{\hat{\eta}}(2,2, \mathbb{Z})$ transformations listed in eqs. (A.11) and (A.12).

For the (symmetric) $\mathbb{T}^{2} / \mathbb{Z}_{2}$ orbifold under consideration, the Narain twist is given by $\Theta=\hat{\Theta}=-\mathbb{1}_{4}$. Hence, all outer automorphisms of the Narain lattice generated by the elements listed in eqs. (A.11) and (A.12) are also outer automorphisms of the $\mathbb{Z}_{2}$ Narain space group. In addition, eq. (A.5) yields translational outer automorphisms $\hat{h}=\left(\mathbb{1}_{4}, T\right) \notin$ $\hat{S}_{\text {Narain }}$ that have to satisfy

$$
(\hat{\Theta}, N) \stackrel{\hat{h}}{\longmapsto}\left(\mathbb{1}_{4},-T\right)(\hat{\Theta}, N)\left(\mathbb{1}_{4}, T\right)=\left(\hat{\Theta}, N-\left(\mathbb{1}_{4}-\hat{\Theta}\right) T\right) \stackrel{!}{\in} \hat{S}_{\text {Narain }} .
$$

Thus, we obtain the condition $\left(\mathbb{1}_{4}-\hat{\Theta}\right) T \in \mathbb{Z}^{4}$ on Narain translations with $T \notin \mathbb{Z}^{4}$. For $\hat{\Theta}=-\mathbb{1}_{4}$ we find that the solutions of this condition can be generated by (cf. appendix B)

$$
T_{1}=\frac{1}{2}\left(\begin{array}{l}
1 \\
0 \\
0 \\
0
\end{array}\right), \quad T_{2}=\frac{1}{2}\left(\begin{array}{l}
0 \\
1 \\
0 \\
0
\end{array}\right), \quad T_{3}=\frac{1}{2}\left(\begin{array}{l}
0 \\
0 \\
1 \\
0
\end{array}\right) \quad \text { and } \quad T_{4}=\frac{1}{2}\left(\begin{array}{l}
0 \\
0 \\
0 \\
1
\end{array}\right) .
$$

\section{A.3 Transformation of $\mathbb{Z}_{2}$ twisted strings}

From eq. (A.16) we find that Narain translations $\hat{h}_{i}=\left(\mathbb{1}_{4}, T_{i}\right) \notin \hat{S}_{\text {Narain }}$ with $T_{i}$ given in eq. (A.17) act on the constructing elements $(\hat{\Theta}, N) \in \hat{S}_{\text {Narain }}$ of $\mathbb{Z}_{2}$ twisted matter fields $\phi_{\left(n_{1}, n_{2}\right)}$ as

$$
(\hat{\Theta}, N) \stackrel{\hat{h}_{i}}{\longmapsto}\left(\hat{\Theta}, N-2 T_{i}\right) \in \hat{S}_{\text {Narain }},
$$

using $\hat{\Theta} T_{i}=-T_{i}$. Note that winding numbers $\left(n_{1}, n_{2}\right)$ and KK numbers $\left(m_{1}, m_{2}\right)$ of $\mathbb{Z}_{2}$ twisted strings with $N=\left(n_{1}, n_{2}, m_{1}, m_{2}\right)^{\mathrm{T}}$ are defined modulo 2 (by considering the conjugacy classes $[\hat{g}]$ of constructing elements $\hat{g} \in \hat{S}_{\text {Narain }}$ ). Consequently, eq. (A.18) shows that $\hat{h}_{1}$ and $\hat{h}_{2}$ increase the winding number $n_{1}$ and $n_{2}$ by one unit, respectively. This confirms the geometrical intuition, illustrated in figure 2: $\hat{h}_{1}$ interchanges the twisted matter fields $\phi_{\left(0, n_{2}\right)}$ and $\phi_{\left(1, n_{2}\right)}$, while $\hat{h}_{2}$ interchanges $\phi_{\left(n_{1}, 0\right)}$ and $\phi_{\left(n_{1}, 1\right)}$. On the other hand, $\hat{h}_{3}$ and $\hat{h}_{4}$ act only on the KK numbers $m_{1}$ and $m_{2}$ of the twisted strings. Hence, each twisted matter field $\phi_{\left(n_{1}, n_{2}\right)}$ is mapped by $\hat{h}_{3}$ and $\hat{h}_{4}$ to itself, possibly times a phase. In summary, these considerations show that the Narain automorphism $\hat{h}_{i}=\left(\mathbb{1}_{4}, T_{i}\right)$ gives rise to a representation $\rho_{4}\left(h_{i}\right)$, for $i=1, \ldots, 4$, as displayed in eqs. (2.11), (2.12a) and (2.12b). 


\section{A.4 Details on mirror symmetry}

Let us consider the constructing elements $\hat{g}=(\hat{\Theta}, N) \in \hat{S}_{\text {Narain }}$ of $\mathbb{Z}_{2}$ twisted strings with $N=\left(n_{1}, n_{2}, m_{1}, m_{2}\right)^{\mathrm{T}}$ and $n_{1}, n_{2}, m_{1}, m_{2} \in\{0,1\}$. These 16 elements are associated with four twisted matter fields $\phi_{\left(n_{1}, n_{2}\right)}$ as follows:

$$
\phi_{\left(n_{1}, n_{2}\right)} \leftrightarrow \hat{g}=(\hat{\Theta}, N) \text { with } \quad N=\left(n_{1}, n_{2}, m_{1}, m_{2}\right)^{\mathrm{T}} \quad \text { and } \quad m_{1}, m_{2} \in\{0,1\} .
$$

Then, according to eq. (A.7) mirror symmetry $\hat{M}$ acts as

$$
(\hat{\Theta}, N) \stackrel{\hat{M}}{\longmapsto}\left(\hat{\Theta}, \hat{M}^{-1} N\right) \in \hat{S}_{\text {Narain }},
$$

where we used $\hat{M}^{-1} \hat{\Theta} \hat{M}=\hat{\Theta}$ for the $\mathbb{Z}_{2}$ Narain twist $\hat{\Theta}=-\mathbb{1}_{4}$, and $\hat{M}$ is given in eq. (A.12). Hence, we obtain

$$
\left(\begin{array}{c}
n_{1} \\
n_{2} \\
m_{1} \\
m_{2}
\end{array}\right) \stackrel{\hat{M}}{\longmapsto}\left(\begin{array}{cccc}
0 & 0 & -1 & 0 \\
0 & 1 & 0 & 0 \\
-1 & 0 & 0 & 0 \\
0 & 0 & 0 & 1
\end{array}\right)\left(\begin{array}{c}
n_{1} \\
n_{2} \\
m_{1} \\
m_{2}
\end{array}\right)=\left(\begin{array}{c}
-m_{1} \\
n_{2} \\
-n_{1} \\
m_{2}
\end{array}\right) .
$$

Using the correspondence between constructing elements of the Narain space group and the twisted matter fields stated in eq. (A.19), we get

$$
\begin{array}{ll}
\phi_{(0,0)} & \stackrel{\hat{M}}{\longmapsto} \alpha_{11} \phi_{(0,0)}+\alpha_{12} \phi_{(1,0)}, \\
\phi_{(1,0)} & \stackrel{\hat{M}}{\longmapsto} \alpha_{21} \phi_{(0,0)}+\alpha_{22} \phi_{(1,0)}, \\
\phi_{(0,1)} \stackrel{\hat{M}}{\longmapsto} \alpha_{33} \phi_{(0,1)}+\alpha_{34} \phi_{(1,1)}, \\
\phi_{(1,1)} \stackrel{\hat{M}}{\longmapsto} \alpha_{43} \phi_{(0,1)}+\alpha_{44} \phi_{(1,1)},
\end{array}
$$

with unknown coefficients $\alpha_{i j}$. Similar to eq. (2.9), this defines the matrix representation of the mirror transformation $\hat{M}$ on twisted matter fields

$$
\tilde{\rho}_{4}(\hat{M}):=\left(\begin{array}{cccc}
\alpha_{11} & \alpha_{12} & 0 & 0 \\
\alpha_{21} & \alpha_{22} & 0 & 0 \\
0 & 0 & \alpha_{33} & \alpha_{34} \\
0 & 0 & \alpha_{43} & \alpha_{44}
\end{array}\right)
$$

where we have used the notation $\tilde{\rho}_{4}(\hat{M})$ (i.e. with a tilde) as it will be redefined at the end of this section.

Moreover, one can analyze the group of outer automorphisms of $\hat{S}_{\text {Narain }}$, especially concerning the translations $T_{i}, i=1, \ldots, 4$, given in eq. (A.17) and mirror symmetry $\hat{M}$, i.e.

$$
\begin{aligned}
& (\hat{M}, 0)\left(\mathbb{1}_{4}, T_{1}\right)\left(\hat{M}^{-1}, 0\right)=\left(\mathbb{1}_{4},-T_{3}\right), \\
& (\hat{M}, 0)\left(\mathbb{1}_{4}, T_{2}\right)\left(\hat{M}^{-1}, 0\right)=\left(\mathbb{1}_{4}, T_{2}\right), \\
& (\hat{M}, 0)\left(\mathbb{1}_{4}, T_{3}\right)\left(\hat{M}^{-1}, 0\right)=\left(\mathbb{1}_{4},-T_{1}\right), \\
& (\hat{M}, 0)\left(\mathbb{1}_{4}, T_{4}\right)\left(\hat{M}^{-1}, 0\right)=\left(\mathbb{1}_{4}, T_{4}\right),
\end{aligned}
$$


where $T_{i}$ are defined up to integers such that $-T_{i} \sim T_{i}$. Let us embed these equations into their action on twisted matter fields

$$
\begin{aligned}
& \tilde{\rho}_{\mathbf{4}}(\hat{M}) \rho_{\mathbf{4}}\left(h_{1}\right) \tilde{\rho}_{\mathbf{4}}(\hat{M})^{-1}=\rho_{\mathbf{4}}\left(h_{3}\right), \\
& \tilde{\rho}_{\mathbf{4}}(\hat{M}) \rho_{\mathbf{4}}\left(h_{2}\right) \tilde{\rho}_{\mathbf{4}}(\hat{M})^{-1}=\rho_{\mathbf{4}}\left(h_{2}\right), \\
& \tilde{\rho}_{\mathbf{4}}(\hat{M}) \rho_{\mathbf{4}}\left(h_{3}\right) \tilde{\rho}_{\mathbf{4}}(\hat{M})^{-1}=\rho_{\mathbf{4}}\left(h_{1}\right), \\
& \tilde{\rho}_{\mathbf{4}}(\hat{M}) \rho_{\mathbf{4}}\left(h_{4}\right) \tilde{\rho}_{\mathbf{4}}(\hat{M})^{-1}=\rho_{\mathbf{4}}\left(h_{4}\right) .
\end{aligned}
$$

This fixes all unknowns $\alpha_{i j}$ except for $\alpha_{11}$,

$$
\tilde{\rho}_{4}(\hat{M})=\alpha_{11}\left(\begin{array}{cccc}
1 & 1 & 0 & 0 \\
1 & -1 & 0 & 0 \\
0 & 0 & 1 & 1 \\
0 & 0 & 1 & -1
\end{array}\right)
$$

and we obtain a representation of mirror transformation that is of order two by setting $\alpha_{11}=\frac{1}{\sqrt{2}}$. However, in order to disentangle the two finite modular groups (associated with the Kähler modulus and the complex structure modulus) we have decided to redefine $\tilde{\rho}_{\mathbf{4}}(\hat{M})$ according to

$$
\rho_{\mathbf{4}}(\hat{M}):=\tilde{\rho}_{\mathbf{4}}(\hat{M}) \rho_{\mathbf{4}}\left(h_{1}\right) \rho_{\mathbf{4}}\left(h_{2}\right) \rho_{\mathbf{4}}\left(h_{3}\right) \rho_{\mathbf{4}}\left(h_{4}\right)=\frac{1}{\sqrt{2}}\left(\begin{array}{cccc}
0 & 0 & -1 & 1 \\
0 & 0 & 1 & 1 \\
1 & -1 & 0 & 0 \\
-1 & -1 & 0 & 0
\end{array}\right),
$$

as stated in eq. (3.6) in section 3. This redefinition is possible, since each transformation $h_{i}$ associated with $\rho_{\mathbf{4}}\left(h_{i}\right)$ in eq. (A.27) belongs to the traditional flavor symmetry that does not affect the moduli and, hence, is valid everywhere in moduli space. Further, the redefinition eq. (A.27) does not alter the physics of the theory because $\rho_{\mathbf{4}}\left(h_{i}\right)$ is a symmetry transformation. Additional details will be given in ref. [33].

\section{B How to classify the outer automorphisms of a space group}

The group of outer automorphisms Out $\left(\hat{S}_{\text {Narain }}\right)$ of a Narain space group $\hat{S}_{\text {Narain }}$ is the key to uncover all discrete symmetries resulting from the orbifold compactification encoded in $\hat{S}_{\text {Narain }}$. Interestingly, there exists a general algebraic construction of $\operatorname{Out}(S)$ for any kind of space group $S$ due to Lutowski, see refs. [35, 36]. In this appendix, we briefly demonstrate its application to the Narain space group of the $\mathbb{T}^{2} / \mathbb{Z}_{2}$ orbifold in order to confirm the results presented in appendix A.2.

The $\mathbb{Z}_{2}$ Narain space group of our $\mathbb{T}^{2} / \mathbb{Z}_{2}$ orbifold is defined as

$$
\hat{S}_{\text {Narain }}=\left\langle\left(\mathbb{1}_{4}, N\right),(\hat{\Theta}, 0) \mid N \in \mathbb{Z}^{4}, \hat{\Theta}=-\mathbb{1}_{4}\right\rangle
$$

in the lattice basis. Then, Lutowski's algorithm states that Out $\left(\hat{S}_{\text {Narain }}\right)$ is given by two factor groups, denoted by $\Xi$ and $\mathrm{H}^{1}\left(\hat{P}, \mathbb{Z}^{4}\right)$. These groups combine semi-directly in the same way as the lattice and the point group combine to define $\hat{S}_{\text {Narain }}$, i.e.

$$
\operatorname{Out}\left(\hat{S}_{\text {Narain }}\right)=\Xi \ltimes \mathrm{H}^{1}\left(\hat{P}, \mathbb{Z}^{4}\right) \text {. }
$$


The constituents of Out $\left(\hat{S}_{\text {Narain }}\right)$ are the so-called stabilizer $\Xi$ of the space group and the first cohomology group $\mathrm{H}^{1}\left(\hat{P}, \mathbb{Z}^{4}\right)$, where $\hat{P}$ is the Narain point group in the lattice basis $\left(\hat{P}=\langle\hat{\Theta}\rangle=\left\{ \pm \mathbb{1}_{4}\right\} \cong \mathbb{Z}_{2}\right.$ in our case). Therefore, one can write an outer automorphism as $\hat{h}=(\hat{\Sigma}, T) \notin \hat{S}_{\text {Narain }}$ with a rotational part $\hat{\Sigma}$ and a translational part $T$ that acts as

$$
\left(\hat{\Theta}^{k}, N\right) \stackrel{\hat{h}}{\longmapsto}(\hat{\Sigma}, T)^{-1}\left(\hat{\Theta}^{k}, N\right)(\hat{\Sigma}, T) \in \hat{S}_{\text {Narain }}
$$

for all $\left(\hat{\Theta}^{k}, N\right) \in \hat{S}_{\text {Narain }}$. By applying Lutowski's algorithm, one observes that $\mathrm{H}^{1}\left(\hat{P}, \mathbb{Z}^{4}\right)$ accounts for pure translations. In detail, one finds

$$
\mathrm{H}^{1}\left(\hat{P}, \mathbb{Z}^{4}\right)=\left\langle T_{i} \mid i=1,2,3,4\right\rangle / \mathbb{Z}^{4} \cong\left(\mathbb{Z}_{2}\right)^{4},
$$

see eq. (A.17). The four generators of this group $T_{i}$ correspond exactly to the transformations $\hat{h}_{i}$ introduced earlier as the geometrical translations for $i=1,2$ and the space group selection rule for $i=3,4$. Acting on the twisted strings of the $\mathbb{Z}_{2}$ orbifold, these outer automorphisms give rise to the traditional flavor symmetry.

The other factor of Out $\left(\hat{S}_{\text {Narain }}\right)$ in eq. (B.2) is the stabilizer $\Xi$, which is governed by the explicit form of the space group. In general, an element of $\Xi$ might admit both, a rotational part $\hat{\Sigma}$ and a translational part $s(\hat{\Sigma})$ such that a general element is given by a so-called roto-translation $(\hat{\Sigma}, s(\hat{\Sigma}))$. Since the $\mathbb{Z}_{2}$ point group $\hat{P}=\langle\hat{\Theta}\rangle$ considered here acts as a pure rotation on the Narain lattice, we can set $s(\hat{\Sigma})=0$ and the algorithm finds the stabilizer group to be

$$
\mathrm{GL}(4, \mathbb{Z}) /\langle\hat{\Theta}\rangle \text {. }
$$

However, we have to impose the physical condition (related to level-matching) that the rotation $\hat{\Sigma}$ has to preserve the Narain metric in the lattice basis $\hat{\eta}$, given in eq. (A.10). This means that $\hat{\Sigma}^{\mathrm{T}} \hat{\eta} \hat{\Sigma}=\hat{\eta}$ has to hold. As defined in eq. (A.9), these rotations form the subgroup $\mathrm{O}_{\hat{\eta}}(2,2, \mathbb{Z})$ of $\mathrm{GL}(4, \mathbb{Z})$. Therefore, the stabilizer group $\Xi$ of the $\mathbb{Z}_{2}$ Narain space group is given by

$$
\Xi \cong \mathrm{O}_{\hat{\eta}}(2,2, \mathbb{Z}) /\langle\hat{\Theta}\rangle .
$$

Its set of elements is generated by $\hat{K}_{\mathrm{S}}, \hat{K}_{\mathrm{T}}, \hat{C}_{\mathrm{S}}, \hat{C}_{\mathrm{T}}, \hat{\Sigma}_{*}$ and $\hat{M}$, as listed in eqs. (A.11) and (A.12), modulo point group transformations with $\hat{\Theta}=-\mathbb{1}_{4}$. This class of outer automorphisms of the Narain space group generates the group of modular transformations of our $\mathbb{T}^{2} / \mathbb{Z}_{2}$ compactification background.

Finally, with the semi-direct product being mediated by the Narain version of the group law in eq. (2.2), the outer automorphisms of the Narain space group reads

$$
\operatorname{Out}\left(\hat{S}_{\text {Narain }}\right)=\left\langle(\hat{\Sigma}, 0),\left(\mathbb{1}_{4}, T_{i}\right) \mid \hat{\Sigma} \in\left\{\hat{K}_{\mathrm{S}}, \hat{K}_{\mathrm{T}}, \hat{C}_{\mathrm{S}}, \hat{C}_{\mathrm{T}}, \hat{\Sigma}_{*}, \hat{M}\right\}, i=1, \ldots, 4\right\rangle / \hat{S}_{\text {Narain }} .
$$

\section{Irreducible representations of twisted matter fields}

The four twisted matter fields $\left(\phi_{(0,0)}, \phi_{(1,0)}, \phi_{(0,1)}, \phi_{(1,1)}\right)^{\mathrm{T}}$ are localized at the four fixed points of the $\mathbb{T}^{2} / \mathbb{Z}_{2}$ orbifold. They transform under both, the traditional flavor symmetry 
$\left(D_{8} \times D_{8}\right) / \mathbb{Z}_{2} \cong[32,49]$, where they transform as an irreducible $\mathbf{4}$, and also under the modular symmetries discussed in section 3 . There, it was found that twisted matter fields do not transform faithfully under $\operatorname{SL}(2, \mathbb{Z})_{T}$ and $\operatorname{SL}(2, \mathbb{Z})_{U}$ but in a four-dimensional unitary representation $\rho_{\mathbf{4}}$ of the respective finite modular groups $S_{3}^{T}$ and $S_{3}^{U}$. Moreover, this representation turns out to be reducible. In detail, by studying the characters of the representation matrices one can show that the four-dimensional representation decomposes i) into irreducible representations of each $S_{3}$ factor as

$$
\mathbf{4}=\mathbf{2} \oplus \mathbf{1} \oplus \mathbf{1}
$$

and ii) into irreducible representations of $S_{3}^{T} \times S_{3}^{U}$ as

$$
\mathbf{4}=(\mathbf{2}, \mathbf{1}) \oplus(\mathbf{1}, \mathbf{2}) .
$$

Moreover, including mirror symmetry, $S_{3}^{T} \times S_{3}^{U}$ is enhanced to [144,115] (cf. eq. (3.11)), where the four twisted matter fields build an irreducible representation 4 . These decompositions can be made explicit by the following orthogonal basis change $B$ :

$$
\left(\begin{array}{l}
\phi_{1} \\
\phi_{2} \\
\phi_{3} \\
\phi_{4}
\end{array}\right):=B\left(\begin{array}{l}
\phi_{(0,0)} \\
\phi_{(1,0)} \\
\phi_{(0,1)} \\
\phi_{(1,1)}
\end{array}\right), \quad \text { where } \quad B:=\left(\begin{array}{cccc}
0 & \frac{-1}{\sqrt{3}} & \frac{-1}{\sqrt{3}} & \frac{-1}{\sqrt{3}} \\
1 & 0 & 0 & 0 \\
0 & \frac{-2}{\sqrt{6}} & \frac{1}{\sqrt{6}} & \frac{1}{\sqrt{6}} \\
0 & 0 & \frac{1}{\sqrt{2}} & \frac{-1}{\sqrt{2}}
\end{array}\right) .
$$

In this basis, the matrix representations, eqs. (3.5)-(3.8), of modular transformations of the four twisted matter fields $\phi_{n}$ are given by $\rho_{\mathbf{4}}^{\prime}(\gamma)=B \rho_{\mathbf{4}}(\gamma) B^{-1}$. They take the following form

$$
\begin{aligned}
& \rho_{4}^{\prime}\left(\hat{K}_{\mathrm{S}}\right)=\left(\begin{array}{cc|cc}
\frac{-1}{2} & \frac{-\sqrt{3}}{2} & 0 & 0 \\
\frac{-\sqrt{3}}{2} & \frac{1}{2} & 0 & 0 \\
\hline 0 & 0 & 1 & 0 \\
0 & 0 & 0 & 1
\end{array}\right), \quad \rho_{4}^{\prime}\left(\hat{K}_{\mathrm{T}}\right)=\left(\begin{array}{cc|cc}
1 & 0 & 0 & 0 \\
0 & -1 & 0 & 0 \\
\hline 0 & 0 & 1 & 0 \\
0 & 0 & 0 & 1
\end{array}\right), \\
& \rho_{\mathbf{4}}^{\prime}\left(\hat{C}_{\mathrm{S}}\right)=\left(\begin{array}{cc|cc}
1 & 0 & 0 & 0 \\
0 & 1 & 0 & 0 \\
\hline 0 & 0 & \frac{-1}{2} & \frac{-\sqrt{3}}{2} \\
0 & 0 & \frac{-\sqrt{3}}{2} & \frac{1}{2}
\end{array}\right), \quad \quad \rho_{\mathbf{4}}^{\prime}\left(\hat{C}_{\mathrm{T}}\right)=\left(\begin{array}{cc|cc}
1 & 0 & 0 & 0 \\
0 & 1 & 0 & 0 \\
\hline 0 & 0 & 1 & 0 \\
0 & 0 & 0 & -1
\end{array}\right),
\end{aligned}
$$

where we indicate the $2 \times 2$ block structure by horizontal and vertical lines, cf. ref. [37]. This proves the $(\mathbf{2}, \mathbf{1}) \oplus(\mathbf{1}, \mathbf{2})$ block structure with respect to $S_{3}^{T} \times S_{3}^{U}$. Furthermore, in this basis it is easy to see that the mirror transformation $\hat{M}$ interchanges $S_{3}^{T}$ and $S_{3}^{U}$, i.e.

$$
\rho_{\mathbf{4}}^{\prime}(\hat{M})=\left(\begin{array}{cc|cc}
0 & 0 & -1 & 0 \\
0 & 0 & 0 & -1 \\
\hline 1 & 0 & 0 & 0 \\
0 & 1 & 0 & 0
\end{array}\right) .
$$

Finally, the irreducible representations in which the twisted matter fields transform under various components of the eclectic flavor symmetry are summarized in table 1 . 


\begin{tabular}{|cccc|c|}
\hline \multicolumn{4}{|c|}{ finite modular symmetry } & traditional flavor symmetry \\
$S_{3}^{T}$ & $S_{3}^{U}$ & $S_{3}^{T} \times S_{3}^{U}$ & $\left(S_{3}^{T} \times S_{3}^{U}\right) \rtimes \mathbb{Z}_{4}^{\hat{M}}$ & $\left(D_{8} \times D_{8}\right) / \mathbb{Z}_{2}$ \\
\hline $\mathbf{2} \oplus \mathbf{1} \oplus \mathbf{1}$ & $\mathbf{2} \oplus \mathbf{1} \oplus \mathbf{1}$ & $(\mathbf{2}, \mathbf{1}) \oplus(\mathbf{1}, \mathbf{2})$ & $\mathbf{4}$ & $\mathbf{4}$ \\
\hline
\end{tabular}

Table 1. Irreducible representations of twisted matter fields $\left(\phi_{(0,0)}, \phi_{(1,0)}, \phi_{(0,1)}, \phi_{(1,1)}\right)^{\mathrm{T}}$ with respect to the various flavor symmetries (in the absence of string oscillator excitations).

Open Access. This article is distributed under the terms of the Creative Commons Attribution License (CC-BY 4.0), which permits any use, distribution and reproduction in any medium, provided the original author(s) and source are credited.

\section{References}

[1] H.P. Nilles, S. Ramos-Sánchez and P.K.S. Vaudrevange, Eclectic Flavor Groups, JHEP 02 (2020) 045 [arXiv: 2001.01736] [inSPIRE].

[2] F. Feruglio, Are neutrino masses modular forms?, in From My Vast Repertoire... : Guido Altarelli's Legacy, A. Levy, S. Forte and G. Ridolfi eds., World Scientific (2018), chapter 12, pp. 227-266 [arXiv:1706.08749] [INSPIRE].

[3] P.P. Novichkov, J.T. Penedo, S.T. Petcov and A.V. Titov, Generalised CP Symmetry in Modular-Invariant Models of Flavour, JHEP 07 (2019) 165 [arXiv: 1905.11970] [INSPIRE].

[4] X.-G. Liu and G.-J. Ding, Neutrino Masses and Mixing from Double Covering of Finite Modular Groups, JHEP 08 (2019) 134 [arXiv:1907.01488] [INSPIRE].

[5] T. Kobayashi, Y. Shimizu, K. Takagi, M. Tanimoto and T.H. Tatsuishi, A $A_{4}$ lepton flavor model and modulus stabilization from $S_{4}$ modular symmetry, Phys. Rev. D 100 (2019) 115045 [Erratum ibid. 101 (2020) 039904] [arXiv: 1909.05139] [INSPIRE].

[6] X.-G. Liu, C.-Y. Yao, B.-Y. Qu and G.-J. Ding, Half-integral weight modular forms and application to neutrino mass models, Phys. Rev. D 102 (2020) 115035 [arXiv:2007.13706] [INSPIRE].

[7] F. Feruglio and A. Romanino, Neutrino Flavour Symmetries, arXiv:1912.06028 [INSPIRE].

[8] H.P. Nilles, S. Ramos-Sánchez and P.K.S. Vaudrevange, Lessons from eclectic flavor symmetries, Nucl. Phys. B 957 (2020) 115098 [arXiv: 2004.05200] [InSPIRE].

[9] M.-C. Chen, S. Ramos-Sánchez and M. Ratz, A note on the predictions of models with modular flavor symmetries, Phys. Lett. B 801 (2020) 135153 [arXiv:1909.06910] [INSPIRE].

[10] A. Baur, H.P. Nilles, A. Trautner and P.K.S. Vaudrevange, Unification of Flavor, CP, and Modular Symmetries, Phys. Lett. B 795 (2019) 7 [arXiv:1901.03251] [InSPIRE].

[11] A. Baur, H.P. Nilles, A. Trautner and P.K.S. Vaudrevange, A String Theory of Flavor and $\mathcal{C P}$, Nucl. Phys. B 947 (2019) 114737 [arXiv:1908.00805] [INSPIRE].

[12] H.P. Nilles, S. Ramos-Sánchez and P.K.S. Vaudrevange, Eclectic flavor scheme from ten-dimensional string theory - I. Basic results, Phys. Lett. B 808 (2020) 135615 [arXiv:2006.03059] [INSPIRE].

[13] T. Kobayashi, H.P. Nilles, F. Plöger, S. Raby and M. Ratz, Stringy origin of non-Abelian discrete flavor symmetries, Nucl. Phys. B 768 (2007) 135 [hep-ph/0611020] [InSPIRE]. 
[14] The GAP Group, GAP - Groups, Algorithms, and Programming, version 4.11 .0 (2020) https://www.gap-system.org.

[15] T. Dent, CP violation and modular symmetries, Phys. Rev. D 64 (2001) 056005 [hep-ph/0105285] [INSPIRE].

[16] H.P. Nilles, S. Ramos-Sánchez and P.K.S. Vaudrevange, Eclectic flavor scheme from ten-dimensional string theory - II. Detailed technical analysis, arXiv:2010.13798 [INSPIRE].

[17] L.J. Dixon, J.A. Harvey, C. Vafa and E. Witten, Strings on Orbifolds, Nucl. Phys. B 261 (1985) 678 [INSPIRE].

[18] L.J. Dixon, J.A. Harvey, C. Vafa and E. Witten, Strings on Orbifolds. 2, Nucl. Phys. B $\mathbf{2 7 4}$ (1986) 285 [INSPIRE].

[19] L.E. Ibáñez, H.P. Nilles and F. Quevedo, Orbifolds and Wilson Lines, Phys. Lett. B 187 (1987) 25 [INSPIRE].

[20] K.S. Narain, New Heterotic String Theories in Uncompactified Dimensions < 10, Phys. Lett. B 169 (1986) 41 [INSPIRE].

[21] K.S. Narain, M.H. Sarmadi and E. Witten, A Note on Toroidal Compactification of Heterotic String Theory, Nucl. Phys. B 279 (1987) 369 [InSPIRE].

[22] S. Groot Nibbelink and P.K.S. Vaudrevange, T-duality orbifolds of heterotic Narain compactifications, JHEP 04 (2017) 030 [arXiv:1703.05323] [INSPIRE].

[23] S. Hamidi and C. Vafa, Interactions on Orbifolds, Nucl. Phys. B 279 (1987) 465 [InSPIRE].

[24] S. Ramos-Sánchez and P.K.S. Vaudrevange, Note on the space group selection rule for closed strings on orbifolds, JHEP 01 (2019) 055 [arXiv: 1811.00580] [INSPIRE].

[25] T. Kobayashi, S. Raby and R.-J. Zhang, Searching for realistic 4d string models with a Pati-Salam symmetry: Orbifold grand unified theories from heterotic string compactification on a $\mathbb{Z}_{6}$ orbifold, Nucl. Phys. B $\mathbf{7 0 4}$ (2005) 3 [hep-ph/0409098] [INSPIRE].

[26] H. Ohki, S. Uemura and R. Watanabe, Modular flavor symmetry on a magnetized torus, Phys. Rev. D 102 (2020) 085008 [arXiv:2003.04174] [INSPIRE].

[27] I. de Medeiros Varzielas, S.F. King and Y.-L. Zhou, Multiple modular symmetries as the origin of flavor, Phys. Rev. D 101 (2020) 055033 [arXiv:1906.02208] [InSPIRE].

[28] S. Kikuchi, T. Kobayashi, S. Takada, T.H. Tatsuishi and H. Uchida, Revisiting modular symmetry in magnetized torus and orbifold compactifications, Phys. Rev. D 102 (2020) 105010 [arXiv : 2005.12642] [INSPIRE].

[29] J. Lauer, J. Mas and H.P. Nilles, Twisted sector representations of discrete background symmetries for two-dimensional orbifolds, Nucl. Phys. B 351 (1991) 353 [inSPIRE].

[30] B. Eick and M. Horn, The construction of finite solvable groups revisited, J. Algebra 408 (2014) 166.

[31] H. Abe, T. Kobayashi, S. Uemura and J. Yamamoto, Loop Fayet-Iliopoulos terms in $T^{2} / Z_{2}$ models: Instability and moduli stabilization, Phys. Rev. D 102 (2020) 045005 [arXiv: 2003.03512] [INSPIRE].

[32] M.-C. Chen, M. Ratz and A. Trautner, Non-Abelian discrete R symmetries, JHEP 09 (2013) 096 [arXiv: 1306.5112] [inSPIRE]. 
[33] A. Baur, M. Kade, H.P. Nilles, S. Ramos-Sánchez and P.K.S. Vaudrevange, in preparation (2020).

[34] M. Baake, B. Gemunden and R. Odingen, Structure and Representations of the Symmetry Group of the Four-dimensional Cube, J. Math. Phys. 23 (1982) 944 [Erratum ibid. 23 (1982) 2595] [INSPIRE].

[35] R. Lutowski, Finite outer automorphism groups of crystallographic groups, Exp. Math. 22 (2013) 456.

[36] L. Charlap, Bieberbach groups and flat manifolds, in Universitext, Springer, New York NY U.S.A. (1986).

[37] H. Ishimori, T. Kobayashi, H. Ohki, Y. Shimizu, H. Okada and M. Tanimoto, Non-Abelian Discrete Symmetries in Particle Physics, Prog. Theor. Phys. Suppl. 183 (2010) 1 [arXiv:1003.3552] [INSPIRE]. 